\title{
The equivalence problem for generic four-dimensional metrics with two commuting Killing vectors
}

\author{
D. Catalano Ferraioli ${ }^{1}$ (D) M. Marvan ${ }^{2}$
}

Received: 21 January 2019 / Accepted: 24 October 2019 / Published online: 6 November 2019 (c) Fondazione Annali di Matematica Pura ed Applicata and Springer-Verlag GmbH Germany, part of Springer Nature 2019

\begin{abstract}
We consider the equivalence problem of four-dimensional semi-Riemannian metrics with the two-dimensional Abelian Killing algebra. In the generic case we determine a semi-invariant frame and a fundamental set of first-order scalar differential invariants suitable for solution of the equivalence problem. Genericity means that the Killing leaves are not null, the metric is not orthogonally transitive (i.e., the distribution orthogonal to the Killing leaves is nonintegrable), and two explicitly constructed scalar invariants $C_{\rho}$ and $\ell_{\mathcal{C}}$ are nonzero. All the invariants are designed to have tractable coordinate expressions. Assuming the existence of two functionally independent invariants, we solve the equivalence problem in two ways. As an example, we invariantly characterize the Van den Bergh metric. To understand the nongeneric cases, we also find all $\Lambda$-vacuum metrics that are generic in the above sense, except that either $C_{\rho}$ or $\ell_{\mathcal{C}}$ is zero. In this way we extend the Kundu class to $\Lambda$-vacuum metrics. The results of the paper can be exploited for invariant characterization of classes of metrics and for extension of the set of known solutions of the Einstein equations.
\end{abstract}

Keywords Differential invariants $\cdot$ Metric equivalence problem $\cdot$ Kundu class

Mathematics Subject Classification $83 \mathrm{C} 20 \cdot 35 \mathrm{Q} 76$

Dedicated to the memory of A. M. Vinogradov

The first author was partially supported by CNPq, Grants 310577/2015-2 and 422906/2016-6. The second author was supported by GAČR under Project P201/12/G028.

$凶$ D. Catalano Ferraioli

diego.catalano@ufba.br

M. Marvan

Michal.Marvan@math.slu.cz

1 Instituto de Matemática e Estatística, Universidade Federal da Bahia, Campus de Ondina, Av. Adhemar de Barros, S/N, Ondina, Salvador, BA CEP 40.170.110, Brazil

2 Mathematical Institute in Opava, Silesian University in Opava, Na Rybníčku 626/1, 74601 Opava, Czech Republic 


\section{Introduction}

Scalar differential invariants have multiple uses in general relativity. Scalar polynomial invariants [7] arise as scalar contractions of $g, R$ and the covariant derivatives $\nabla R, \ldots, \nabla^{m} R$. Besides being a tool to detect true singularities irremovable by coordinate transformations, scalar differential invariants provide a basis for solving the equivalence problem, i.e., the problem of classifying spacetime metrics with respect to local isometries. Scalar differential invariants can, in principle, solve the equivalence problem except for metrics of the Kundt class [9], but not in an effective way. Here the Cartan-Karlhede invariants, see [16,17] or [35, Ch. 9], come to the rescue. The Cartan-Karlhede invariants, defined as components of the Riemann tensor and its covariant derivatives with respect to suitably chosen frames, lie in the heart of a workable algorithm to decide about equivalence of space-time metrics $[3,18,32]$. Another useful application is that of finding solutions of Einstein's equations by imposing additional invariant constraints $[6,23,24,33]$.

All invariants mentioned so far started at the second order, a strict lower bound for scalar invariants of metrics [40]. To enable first-order metric invariants, one would have to reduce the pseudogroup of diffeomorphisms. One important case when this is easily done is when the metric has Killing fields. The semi-Riemannian manifold then becomes a submersion in a natural way and instead of the equivalence of spacetimes we can consider the equivalence of the semi-Riemannian submersions. This allows the first-order invariants to appear, while not impeding the solution of the equivalence problem, if the submersion is taken with respect to the full Killing algebra.

More precisely, in the case of a semi-Riemannian manifold $(\mathcal{M}, \mathbf{g})$ with the Killing algebra $\mathfrak{K i l l}(\mathbf{g})=\mathcal{G}$, we consider the semi-Riemannian submersion $\mathcal{M} \rightarrow \mathcal{M} / G$, where $\mathcal{M} / G$ is the orbit space of the Lie group $G$ of the transformations generated by $\mathcal{G}$ on $\mathcal{M}$. Obviously, two semi-Riemannian manifolds are isometric if and only if the corresponding semi-Riemannian submersion structures are isomorphic.

In the present paper, we apply the above scheme to the particular class, denoted as $G_{2} I$ (cf. [35, Ch. 17]), of four-dimensional semi-Riemannian metrics whose Killing algebra is Abelian and two dimensional. For generic metrics of this kind, we obtain a fundamental system of functionally independent scalar differential invariants and solve the problem of equivalence. This fundamental system consists of six functionally independent first-order scalar differential invariants. They admit very simple explicit expressions in terms of metric coefficients, in sharp contrast to the curvature invariants mentioned above.

We have chosen the class $G_{2} I$ because it is rather rich in explicit solutions of Einstein equations, especially in the orthogonally transitive subcase (see Sect. 2) when the metric can be written in a block diagonal form and the vacuum, electro-vacuum and some other Einstein equations are integrable in the sense of soliton theory, see [1,11,19,35,36] and references therein. At the same time, only a handful of orthogonally intransitive metrics are known (e.g., [13,20,37-39]). Looking for non-generic cases, one can often obtain explicit solutions. In this paper we just characterize metrics with vanishing invariants $C_{\rho}$ or $\ell_{\mathcal{C}}$. For $C_{\rho}=0$ we find that all $\Lambda$-vacuum metrics are $p p$-waves, whereas for $\ell_{\mathcal{C}}=0$ (the Kundu class) we obtain new explicit $\Lambda$-vacuum solutions.

The paper is organized as follows: In Sect. 2 we introduce the Lie pseudogroup $\mathfrak{G}$ acting on four-dimensional semi-Riemannian manifolds of class $G_{2} I$. In Sect. 3 we describe the infinitesimal generators of $\mathfrak{G}_{\tau}$, the natural extension of $\mathfrak{G}$ to the bundle $\tau$ of metrics, and determine the number of functionally independent differential invariants of jet orders 0,1 , 2. In Sect. 4 we study the orbit space $\mathcal{S}=\mathcal{M} / G_{2}$ and the induced orbit metric $\tilde{\mathbf{g}}$. In Sect. 5 
we introduce a maximal set of 6 generically functionally independent scalar differential invariants $C_{\rho}, C_{\chi}, Q_{\chi}, Q_{\gamma}, \ell_{\mathcal{C}},\left(\Theta_{\mathrm{I}}\right)^{2}$ of the first order. In the generic case, when $C_{\rho}$ and $\ell_{\mathcal{C}}$ do not vanish, we also provide a semi-invariant orthogonal frame. In Sect. 6, we provide a maximal set of 20 generically functionally independent scalar differential invariants of the second order. In Sect. 7, we derive the $\Lambda$-vacuum Einstein equations for $G_{2} I$-metrics, and find their solutions in the two non-generic cases mentioned above. In Sect. 8, we find functionally independent invariants on solutions to the $\Lambda$-vacuum Einstein equations. Finally, in Sect. 9, we address the equivalence problem of $G_{2}$-metrics in the generic case.

For reader's convenience, we provide a list of symbols used throughout the paper, except those limited to the section where they have been defined.

Symbols defined in Sect. 2

$\mathcal{M}$
$\mathcal{G}_{2}$
$G_{2}$
$\mathbf{g}$
$\mathfrak{K i l l}(\mathbf{g})$
$G_{2} I$
$\Xi$
$\xi_{(1)}, \xi_{(2)}$
$\Xi^{\perp}$
$\mathcal{S}$
$\pi: \mathcal{M} \rightarrow \mathcal{S}$
$\left\{t^{1}, t^{2}, z^{1}, z^{2}\right\}$
$\tau: E \rightarrow \mathcal{M}$
$\tau_{m}: J^{m} \tau \rightarrow \mathcal{M}$
$\mathbf{h}=h_{i j} d z^{i} d z^{j}$
$H=\left(h_{i j}\right)$
$\mathfrak{G}^{\mathfrak{G}_{\tau}, \mathfrak{G}_{\tau}^{(k)}}$

Symbols defined in Sect. 3 $\mid \mathbf{g}$

Symbols defined in Sect. 4

$\tilde{\mathbf{g}}=\tilde{g}_{i j} d t^{i} d t^{j}$

Symbols defined in Sect. 5.1

$$
\begin{aligned}
& \sigma=d \ln (\operatorname{det} \mathbf{h}) \\
& \rho, \chi, \gamma \\
& C_{\rho}, C_{\chi}, Q_{\chi}, Q_{\gamma} \\
& \mathcal{X} \text { and } \mathcal{X}^{\perp}
\end{aligned}
$$

a four-dimensional semi-Riemannian manifold; the two-dimensional Abelian Lie algebra; the two-dimensional Abelian Lie group; a metric of class $G_{2} I$ on $\mathcal{M}$; the algebra of Killing fields of $(\mathcal{M}, \mathbf{g})$; the class of four-dimensional semi-Riemannian metrics with $\mathfrak{K i l l}(\mathbf{g})=\mathcal{G}_{2}$;

the integrable two-dimensional distribution on $\mathcal{M}$ determined by $\mathcal{G}_{2}$;

fixed linearly independent Killing fields, local generators of $\Xi$;

the orthogonal complement of $\Xi$;

the two-dimensional orbit space $\mathcal{M} / G_{2}$;

the natural Riemannian submersion;

a system of local adapted coordinates on $\mathcal{M}$; by definition $\xi_{(i)}=\partial_{z^{i}}$ and $\left\{t^{1}, t^{2}\right\}$ are local coordinates on $\mathcal{S}$;

the bundle of metrics;

the $m$ th order jet bundle of sections of $\tau$;

the restriction of $\mathbf{g}$ to the leaves of $\Xi$,

the matrix composed of $h_{i j}$;

the Lie pseudogroup of adapted-coordinates transformations on $\mathcal{M}$;

the natural extension of $\mathfrak{G}$ to $\tau$ and $J^{k} \tau$, respectively;

restriction of a tensor from $J^{\infty} \tau$ to a prolongation of the section of $\tau$ corresponding to $\mathbf{g}$.

the induced metric tensor on $\mathcal{S}$, referred to as the orbit metric.

an invariant 1-form defined in terms of $\mathbf{h}$;

invariant symmetric $(0,2)$-tensors defined in terms of $\mathbf{h}$ and $\tilde{\mathbf{g}}$;

first-order scalar differential invariants;

$\pi_{1}$-relative semi-invariant orthogonal fields on $\mathcal{S}$; 
$\pm_{\tilde{\mathbf{g}}}=\operatorname{sgn} \operatorname{det} \tilde{\mathbf{g}}$ and

$\pm_{\mathbf{h}}=\operatorname{sgn} \operatorname{det} \mathbf{h}=\operatorname{sgn} \operatorname{det} H$

signums.

Symbols defined in Sect. 5.2

c

type $(1,2)$ Ehresmann curvature tensor of the Riemannian submersion $\pi$;

$\mathcal{C}$

the curvature vector field of the Riemannian submersion $\pi$;

$\mathcal{H}$

the mean curvature vector field of the Riemannian submersion $\pi$;

$\left\{\mathcal{C}, \mathcal{C}^{\perp}\right\}$ resp. $\left\{\mathcal{H}, \mathcal{H}^{\perp}\right\}$

$\ell_{\mathcal{C}}$

semi-invariant orthogonal frames in $\Xi$ resp. $\Xi^{\perp}$;

the squared length $\mathbf{g}(\mathcal{C}, \mathcal{C})$ of the curvature vector;

$\Theta_{\mathrm{I}}$ another first-order scalar invariant.

\section{The pseudogroup and the metric}

Let $\mathcal{G}_{2}$ denote the two-dimensional Abelian Lie algebra. Let $\mathcal{M}$ be a four-dimensional manifold, endowed with a semi-Riemannian metric $\mathbf{g}$ such that the algebra $\mathfrak{K i l l}(\mathbf{g})$ of Killing vector fields of $\mathbf{g}$ is $\mathcal{G}_{2}$. In particular, there are no Killing vectors outside $\mathcal{G}_{2}$. We denote by $\Xi$ the vector distribution generated on $\mathcal{M}$ by the Killing fields of $\mathcal{G}_{2}$. The two-dimensional integral submanifolds of $\Xi$ are called the Killing leaves. Throughout the paper, we assume that the Killing leaves are non-null. By $G_{2}$ we denote the Lie group of transformations generated by $\mathcal{G}_{2}$ on $\mathcal{M}$. We also assume that the orbit space $\mathcal{S}=\mathcal{M} / G_{2}$ is a two-dimensional manifold, with $\pi: \mathcal{M} \rightarrow \mathcal{S}$ being the natural projection.

Let $\Xi^{\perp}$ denote the distribution orthogonal to the Killing leaves. The case when $\Xi^{\perp}$ is integrable will be referred to as the orthogonally transitive case; otherwise, it will be referred to as the orthogonally intransitive case.

One can always choose local coordinates $\left\{t^{1}, t^{2}, z^{1}, z^{2}\right\}$ on $\mathcal{M}$ such that:

(A) $\mathcal{G}_{2}$ is generated by the coordinate vector fields $\xi_{(i)}=\partial / \partial z^{i}, i=1,2$;

(B) the leaves of $\Xi$ are the surfaces characterized by the constancy of $t^{1}$ and $t^{2}$.

We refer to such a kind of coordinates $\left\{t^{1}, t^{2}, z^{1}, z^{2}\right\}$ as local adapted coordinates. In adapted coordinates, the metric $\mathbf{g}$ takes the form

$$
\mathbf{g}=b_{i j}\left(t^{1}, t^{2}\right) d t^{i} d t^{j}+2 f_{i k}\left(t^{1}, t^{2}\right) d t^{i} d z^{k}+h_{k l}\left(t^{1}, t^{2}\right) d z^{k} d z^{l},
$$

with $b_{21}=b_{12}$ and $h_{21}=h_{12}$.

Let $\mathbf{h}$ denote the restriction of the metric $\mathbf{g}$ to the individual leaves of $\Xi$. By condition (B), $\mathbf{h}=h_{k l}\left(t^{1}, t^{2}\right) d z^{k} d z^{l}$. Let $H$ denote the $2 \times 2$ symmetric matrix $H=\left(h_{i j}\right)$ with elements

$$
h_{k l}=\mathbf{g}\left(\xi_{(k)}, \xi_{(l)}\right)=\mathbf{g}_{a b} \xi_{(k)}^{a} \xi_{(l)}^{b} .
$$

In view of the assumption that $\Xi$ are not null, (see the beginning of this section), det $\mathbf{h}=$ det $H \neq 0$ everywhere.

In the orthogonally transitive case, the leaves of $\Xi^{\perp}$ can be identified with surfaces $z^{1}=$ const, $z^{2}=$ const, which means that the metric can be put in the block diagonal form with $f_{i k}=0$.

We introduce the Lie pseudogroup $\mathfrak{G}$ of transformations of adapted coordinates now. By definition, $\mathfrak{G}$-transformations are coordinate transformations $\bar{t}^{i}=\bar{t}^{i}\left(t^{1}, t^{2}, z^{1}, z^{2}\right), \bar{z}^{i}=$ $\bar{z}^{i}\left(t^{1}, t^{2}, z^{1}, z^{2}\right)$ which preserve (A) and (B), i.e., such that $\mathcal{G}_{2}$ is generated by $\partial / \partial \bar{z}^{i}, i=1,2$, and the leaves of $\Xi$ are surfaces characterized by the constancy of $\bar{t}^{1}$ and $\bar{t}^{2}$. 
Proposition 1 The Lie pseudogroup $\mathfrak{G}$ is formed by transformations $P: \mathcal{M} \rightarrow \mathcal{M}$ which in adapted coordinates have the form

$$
\bar{t}^{i}=\phi^{i}\left(t^{1}, t^{2}\right), \quad \bar{z}^{i}=\alpha_{j}^{i} z^{j}+\psi^{i}\left(t^{1}, t^{2}\right),
$$

where $\phi^{i}\left(t^{1}, t^{2}\right)$ and $\psi^{i}\left(t^{1}, t^{2}\right)$ are arbitrary differentiable functions satisfying

$$
J_{\phi}=\left|\begin{array}{ll}
\partial_{t^{1}} \phi^{1} & \partial_{t^{2}} \phi^{1} \\
\partial_{t^{1}} \phi^{2} & \partial_{t^{2}} \phi^{2}
\end{array}\right| \neq 0,
$$

and $\alpha_{j}^{i} \in \mathbb{R}$, with $\left(\alpha_{j}^{i}\right) \in \mathrm{GL}(2, \mathbb{R})$.

Infinitesimal generators of $\mathfrak{G}$ have the form

$$
U=\Phi^{i}\left(t^{1}, t^{2}\right) \frac{\partial}{\partial t^{i}}+\left(A_{l}^{k} z^{l}+\Psi^{k}\left(t^{1}, t^{2}\right)\right) \frac{\partial}{\partial z^{k}},
$$

where $\Phi^{i}\left(t^{1}, t^{2}\right)$ and $\Psi^{k}\left(t^{1}, t^{2}\right)$ are arbitrary differentiable functions and $A_{l}^{k} \in \mathbb{R}$ are arbitrary constants.

In particular, $\mathfrak{G}$ can be decomposed as

$$
\mathfrak{G}=\mathfrak{G}_{+,+} \cup \mathfrak{G}_{+,-} \cup \mathfrak{G}_{-,+} \cup \mathfrak{G}_{-,-},
$$

where $\mathfrak{G}_{\epsilon_{1}, \epsilon_{2}}$ are the connected components, with $\epsilon_{1}=\operatorname{sgn} J_{\phi}$ and $\epsilon_{2}=\operatorname{sgn}\left(\operatorname{det} \alpha_{j}^{i}\right)$.

Proof Under a $\mathfrak{G}$-transformation $\partial / \partial z^{j}=\alpha_{j}^{i} \partial / \partial \bar{z}^{i}$, with $\left(\alpha_{j}^{i}\right) \in \operatorname{GL}(2, \mathbb{R})$. Since $\partial / \partial z^{j}=$ $\left(\partial \bar{z}^{i} / \partial z^{j}\right) \partial / \partial \bar{z}^{i}+\left(\partial \bar{t}^{i} / \partial z^{j}\right) \partial / \partial \bar{t}^{i}$, one gets $\partial \bar{z}^{i} / \partial z^{j}=\alpha_{j}^{i}, \partial \bar{t}^{i} / \partial z^{j}=0$. Hence, the $\mathfrak{G}$-transformations have the required form.

Next, a vector field $U=T^{i}(t, z) \partial / \partial t^{i}+Z^{k}(t, z) \partial / \partial z^{k}$ is an infinitesimal generator of $\mathfrak{G}$ iff $U$ is an infinitesimal symmetry of the Lie algebra generated by $\xi_{(1)}=\partial / \partial z^{1}$ and $\xi_{(2)}=\partial / \partial z^{2}$. Therefore, $\left[\partial / \partial z^{l}, U\right]=A_{l}^{k} \partial / \partial z^{k}$, with $A_{l}^{k}$ arbitrary constants. Hence, $\partial T^{i}(t, z) / \partial z^{l}=0, \partial Z^{k}(t, z) / \partial z^{l}=A_{l}^{k}$, and the statement readily follows.

It is worth noting here that under $\mathfrak{G}$-transformations (2), $\mathbf{g}$ transforms to

$$
\overline{\mathbf{g}}=\bar{b}_{m n}\left(\bar{t}^{1}, \bar{t}^{2}\right) \mathrm{d} \bar{t}^{m} \mathrm{~d} \bar{t}^{n}+2 \bar{f}_{m r}\left(\bar{t}^{1}, \bar{t}^{2}\right) \mathrm{d} \bar{t}^{m} \mathrm{~d} \bar{z}^{r}+\bar{h}_{r s}\left(\bar{t}^{1}, \bar{t}^{2}\right) \mathrm{d} \bar{z}^{r} \mathrm{~d} \bar{z}^{s},
$$

with

$$
\begin{aligned}
b_{i j} & =\bar{b}_{m n} \frac{\partial \phi^{m}}{\partial t^{i}} \frac{\partial \phi^{n}}{\partial t^{j}}+2 \bar{f}_{m r} \frac{\partial \phi^{m}}{\partial t^{i}} \frac{\partial \psi^{r}}{\partial t^{j}}+\bar{h}_{r s} \frac{\partial \psi^{r}}{\partial t^{i}} \frac{\partial \psi^{s}}{\partial t^{j}}, \\
f_{i k} & =\bar{f}_{m r} \alpha_{k}^{r} \frac{\partial \phi^{m}}{\partial t^{i}}+\bar{h}_{r s} \alpha_{k}^{r} \frac{\partial \psi^{s}}{\partial t^{i}}, \\
h_{k l} & =\bar{h}_{r s} \alpha_{k}^{r} \alpha_{l}^{s} .
\end{aligned}
$$

In particular

$$
\operatorname{det} \overline{\mathbf{g}}=\left(\operatorname{det} \alpha_{j}^{i}\right)^{2}\left(J_{\phi}\right)^{2} \operatorname{det} \mathbf{g} \neq 0 .
$$

Semi-Riemannian metrics g, with $\mathfrak{K i l l}(\mathbf{g})=\mathcal{G}_{2}$ and non-null Killing leaves, locally described by (1), form a subbundle of the bundle of symmetric $(0,2)$-tensors $S^{2}\left(T^{*} \mathcal{M}\right) \rightarrow$ $\mathcal{M}$. We denote the subbundle by $\tau: E \rightarrow \mathcal{M}$.

Proposition 2 The pseudogroup $\mathfrak{G}$ naturally extends to the bundle $\tau: E \rightarrow \mathcal{M}$ of metrics of the form (1) on $\mathcal{M}$.

Proof See formulas (6) and (7).

The extension of $\mathfrak{G}$ to $\tau$ will be denoted by $\mathfrak{G}_{\tau}$. 


\section{Pseudogroup prolongation and differential invariants}

In view of Proposition 2, the classification problem for metrics with an Abelian twodimensional Killing algebra $\mathcal{G}_{2}$ reduces to identifying orbits of the action of $\mathfrak{G}_{\tau}$ on the vector bundle $\tau: E \rightarrow \mathcal{M}$ of metrics $\mathbf{g}$ of the form (1); indeed, these orbits consist of mutually equivalent metrics.

Following Lie's classical method, the classification problem for these metrics can be solved by using a sufficient number of independent scalar differential invariants of $\mathfrak{G}_{\tau}$. These invariants are defined to be functions on the jet prolongations $J^{m} \tau, m=0,1,2, \ldots$, that are invariant with respect to the action of the corresponding prolonged pseudogroups $\mathfrak{G}_{\tau}^{(m)}$.

The problem of finding the $m$-th order scalar differential invariants becomes linear if written in terms of the infinitesimal action of $\mathfrak{G}_{\tau}^{(m)}$ on $J^{m} \tau$. This fact is at the heart of Lie's infinitesimal method of computing differential invariants and also permits a simple determination of the dimensions $N_{m}$ of the orbit spaces $J^{m} \tau / \mathfrak{G}_{\tau}^{(m)}$ for $m=0,1,2, \ldots$

Proposition 3 By using the coordinate representation (1), the pseudogroup $\mathfrak{G}_{\tau}$ is infinitesimally generated by vector fields

$$
\begin{aligned}
U^{\tau}= & \Phi^{i} \frac{\partial}{\partial t^{i}}+\left(A_{l}^{k} z^{l}+\Psi^{k}\right) \frac{\partial}{\partial z^{k}} \\
& -\left(b_{i s} \frac{\partial \Phi^{s}}{\partial t^{j}}+f_{i s} \frac{\partial \Psi^{s}}{\partial t^{j}}+b_{j s} \frac{\partial \Phi^{s}}{\partial t^{i}}+f_{j s} \frac{\partial \Psi^{s}}{\partial t^{i}}\right) \frac{\partial}{\partial b_{i j}} \\
& -\left(f_{s k} \frac{\partial \Phi^{s}}{\partial t^{i}}+h_{s k} \frac{\partial \Psi^{s}}{\partial t^{i}}+f_{i s} A_{k}^{s}\right) \frac{\partial}{\partial f_{i k}}-\left(h_{k s} A_{l}^{s}+h_{s l} A_{k}^{s}\right) \frac{\partial}{\partial h_{k l}} .
\end{aligned}
$$

where $\Phi^{i}, \Psi^{i}, A_{l}^{k}$ are as in Proposition 1, formula (4).

Proof Since $U^{\tau}$ projects to $U$, it has the form

$$
U^{\tau}=\Phi^{i}(t) \frac{\partial}{\partial t^{i}}+\left(A_{l}^{k} z^{l}+\Psi^{k}(t)\right) \frac{\partial}{\partial z^{k}}+B_{i j} \frac{\partial}{\partial b_{i j}}+F_{i k} \frac{\partial}{\partial f_{i k}}+H_{k l} \frac{\partial}{\partial h_{k l}},
$$

with $B_{i j}, F_{i k}$ and $H_{k l}$ differentiable functions of $t^{1}, t^{2}, z^{1}, z^{2}$ and $f_{i j}, b_{i j}, h_{k l}$. Then, (9) follows by imposing the Lie invariance condition $L_{U^{\tau}}(\mathbf{g})=0$.

Recall that $J^{0} \tau=\tau$ and that the formal derivatives of $b_{i j}, f_{i k}$ and $h_{k l}$ of orders $m=$ $0,1,2, \ldots$ with respect to $t^{1}, t^{2}$, can serve as coordinates along the fibers of $J^{m} \tau$ in an obvious way. We denote such coordinates as $b_{i j, \mathbf{I}}, f_{i j, \mathbf{I}}$ and $h_{k l, \mathbf{I}}$, for any symmetric multi-index I when $m>1$, and $b_{i j, s}, f_{i j, s}$ and $h_{k l, s}$ when $m=1$.

Prolongation formulas of $U^{\tau}$ to $U^{J^{m} \tau}$ on $J^{m} \tau, m=1,2, \ldots$, are well known [2,27,29]. Alternatively, from the commutator

$$
\left[\frac{\partial}{\partial t^{s}}, U\right]=\frac{\partial \Phi^{j}}{\partial t^{s}} \frac{\partial}{\partial t^{j}}+\left(\frac{\partial A_{l}^{k}}{\partial t^{s}} z^{l}+\frac{\partial \Psi^{k}}{\partial t^{s}}\right) \frac{\partial}{\partial z^{k}}
$$

valid on the base manifold $\mathcal{S}$, one can infer the relations

$$
\left[U^{J^{\infty} \tau}, D_{s}\right]=-\left(\partial \Phi^{1} / \partial t^{s}\right) D_{1}-\left(\partial \Phi^{2} / \partial t^{s}\right) D_{2}
$$

on $J^{\infty} \tau$, where

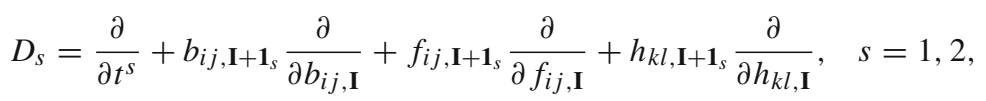


denote the usual total derivatives and I stands for an arbitrary symmetric multi-index. These relations reflect the way how the action on metric coefficients extends to the action on derivatives thereof. Thus, for any symmetric multi-index I of order $m$, we have

$$
U^{J^{m+1} \tau}\left(b_{i j, \mathbf{I}+\mathbf{1}_{s}}\right)=D_{s}\left(U^{J^{m} \tau}\left(b_{i j, \mathbf{I}}\right)\right)-\frac{\partial \Phi^{1}}{\partial t^{s}} b_{i j, \mathbf{I}+\mathbf{1}_{1}}-\frac{\partial \Phi^{2}}{\partial t^{s}} b_{i j, \mathbf{I}+\mathbf{1}_{2}},
$$

and analogously for $f_{i k}$ and $h_{k l}$.

Now, scalar differential invariants can be identified with functions on $J^{\infty} \tau$ invariant with respect to the fields $U^{J^{\infty}} \tau$. These invariants form a commutative associative $\mathbb{R}$-algebra, which can be thought of as algebra of functions on the orbit space $J^{\infty} \tau / \mathfrak{G}_{\tau}^{(\infty)}$.

Proposition 4 In the orthogonally intransitive case, the generic dimension $N_{m}$ of the orbit space $J^{m} \tau / \mathfrak{G}_{\tau}^{(m)}$ for $m=0,1,2$ is given by

$$
\begin{array}{r|rrr}
m & 0 & 1 & 2 \\
\hline N_{m} & 0 & 6 & 20
\end{array}
$$

In the orthogonally transitive case, the generic dimension $N_{m}$ of the orbit space $J^{m} \tau / \mathfrak{G}_{\tau}^{(m)}$ for $m=0,1,2$ is given by

\begin{tabular}{r|rrr}
$m$ & 0 & 1 & 2 \\
\hline$N_{m}$ & 0 & 4 & 14
\end{tabular}

Proof Using the infinitesimal generators of $\mathfrak{G}_{\tau}^{(m)}$, obtained by prolonging (9) to $J^{m} \tau$, and taking the coefficients at $\Phi^{i}, A_{l}^{k}, \Psi^{k}$ and their derivatives, we obtain a compatible linear system for $m$ th order invariants, $m=0,1,2$. The dimensions of the solution spaces are the dimensions sought. The orthogonally transitive case has been computed in [25].

Of course, there may exist singular strata in $J^{\infty} \tau / \mathfrak{G}_{\tau}^{(\infty)}$ where the maximal number of functionally independent scalar differential invariants is lower than $N_{m}$.

Remark 1 Proposition 4 refers to scalar differential invariants as functions on the jet space $J^{\infty} \tau$. If such a function, say $F$, is evaluated for a particular metric $\mathbf{g}$, then it becomes a function on the orbit space $\mathcal{S}$, which we shall denote as $\left.F\right|_{\mathbf{g}}$ (formally $\left.F\right|_{\mathbf{g}}=F \circ j^{\infty} \sigma_{\mathbf{g}}$, where $j^{\infty}$ denotes a jet prolongation of a section of the bundle $\tau$ and $\sigma_{\mathbf{g}}$ is the section associated with $\mathbf{g}$ ), hence another interpretation of scalar differential invariants as functions on $\mathcal{S}$. Both interpretations are natural and important. For instance, the order of an invariant can only be seen in the context of jet spaces, while the most natural way to construct an invariant consists in combining various invariant geometric constructions on $\mathcal{S}$ [2]. We shall often use one and the same notation with both interpretations and omit the symbol $\left.\right|_{\mathrm{g}}$. However, one should bear in mind that independence of functions on $\mathcal{S}$ is very different from that on $J^{\infty} \tau$. The maximal number of independent functions is two on $\mathcal{S}$, and unlimited on $J^{\infty} \tau$.

\section{Orbit metric}

We found it very convenient to rewrite the metric in the form

$$
\mathbf{g}=\tilde{g}_{i j} d t^{i} d t^{j}+h_{k l}\left(d z^{k}+f_{i}^{k} d t^{i}\right)\left(d z^{l}+f_{j}^{l} d t^{j}\right),
$$

where

$$
\tilde{g}_{i j}=b_{i j}-f_{i k} f_{j l} h^{k l}, \quad f_{j}^{k}=f_{j s} h^{s k}
$$


and $h^{k l}$ denote the elements of the inverse matrix $H^{-1}$. Notice that relations (11) directly connect components of (1) to those of (10).

In terms of variables $\tilde{g}_{i j}, f_{i}^{k}, h_{k l}$, expression (9) for $U^{\tau}$ simplifies to

$$
\begin{aligned}
U^{\tau}= & \Phi^{i} \frac{\partial}{\partial t^{i}}+\left(A_{l}^{k} z^{l}+\Psi^{k}\right) \frac{\partial}{\partial z^{k}}-\left(\tilde{g}_{i s} \frac{\partial \Phi^{s}}{\partial t^{j}}+\tilde{g}_{j s} \frac{\partial \Phi^{s}}{\partial t^{i}}\right) \frac{\partial}{\partial \tilde{g}_{i j}} \\
& +\left(f_{i}^{s} A_{s}^{k}-\frac{\partial \Psi^{k}}{\partial t^{i}}-f_{s}^{k} \frac{\partial \Phi^{s}}{\partial t^{i}}\right) \frac{\partial}{\partial f_{i}^{k}}-\left(A_{l}^{s} h_{k s}+A_{k}^{s} h_{s l}\right) \frac{\partial}{\partial h_{k l}} .
\end{aligned}
$$

An important advantage of (10) is that $\tilde{\mathbf{g}}=\tilde{g}_{i j} d t^{i} d t^{j}$ defines a natural metric on the orbit space $\mathcal{S}$ such that

$$
\tilde{\mathbf{g}}(X, Y)=\mathbf{g}(X, Y)-h^{k l} \mathbf{g}\left(\xi_{(k)}, X\right) \mathbf{g}\left(\xi_{(l)}, Y\right),
$$

for any pair of vector fields $X, Y$ on $\mathcal{S}$.

Proposition 5 (Geroch [14]) The (0,2)-tensor field $\tilde{\mathbf{g}}$ defines a metric tensor on the orbit space $\mathcal{S}=\mathcal{M} / G_{2}$.

Proof The components of $\tilde{\mathbf{g}}$ depend only on $\left(t^{1}, t^{2}\right)$ and, since $\tilde{\mathbf{g}}\left(\xi_{(i)},-\right)=0$ for $i=1,2$, we have $\tilde{\mathbf{g}}(X,-)=0$ for every vector field $X \in \Xi$. Therefore, $\tilde{\mathbf{g}}$ is a well-defined $(0,2)$ tensor on the two-dimensional orbit space $\mathcal{S}=\mathcal{M} / G_{2}$, and

$$
\tilde{g}_{i j}=b_{i j}-f_{i k} f_{j l} h^{k l}, \quad i, j=1, \ldots, 2 .
$$

Moreover, by (10), it is easily checked that

$$
\operatorname{det} \tilde{\mathbf{g}}=\frac{\operatorname{det} \mathbf{g}}{\operatorname{det} \mathbf{h}} \text {. }
$$

Hence, $\tilde{\mathbf{g}}$ is non-degenerate and defines a metric on $\mathcal{S}$.

Explicit expressions of differential invariants of $\mathbf{g}$ are relatively simple in terms of $\tilde{g}_{i j}, f_{i}^{k}, h_{k l}$, whereas they swell in $b_{i j}, f_{i k}, h_{k l}$.

\section{First-order invariants}

According to Proposition 4 , on $J^{1} \tau$ there are at most 4 functionally independent scalar invariants in the orthogonal transitive case (when $\Xi^{\perp}$ is integrable), and at most 6 functionally independent invariants in the orthogonally intransitive case (when $\Xi^{\perp}$ is not integrable). Such a maximal system of functionally independent invariants generates the whole algebra of differential invariants of the first order, since any first-order scalar differential invariant must be a function of them. In this section we provide an explicit construction of a maximal system of 6 functionally independent scalar invariants for the orthogonally intransitive case, which extends the already known [25] maximal system $I_{1}=C_{\rho}, I_{2}=C_{\chi}, I_{3}=Q_{\chi}$, $I_{4}=Q_{\gamma}$ of invariants for the orthogonally transitive case.

\subsection{Scalar invariants $C_{\rho}, C_{\chi}, Q_{\chi}, Q_{\gamma}$ and the semi-invariant orthogonal frame $\left\{\mathcal{X}, \mathcal{X}^{\perp}\right\}$ on $\mathcal{S}$}

The first-order invariants presented in this subsection essentially coincide with those of [25] except that the metric coefficients $g_{i j}$ of [25] have been replaced with the coefficients of the orbit space metric $\tilde{\mathbf{g}}_{i j}$. 
Lemma 1 For any metric $\mathbf{g}$ of the form (10), the pseudogroup action leaves invariant the 1 -form

$$
\sigma=d \ln (\operatorname{det} \mathbf{h})=\frac{d(\operatorname{det} \mathbf{h})}{\operatorname{det} \mathbf{h}}
$$

and the symmetric $(0,2)$-tensors

$$
\rho=\sigma^{2}, \quad \chi=\frac{1}{(\operatorname{det} \mathbf{h})}\left(d h_{11} d h_{22}-d h_{12} d h_{12}\right) .
$$

Proof Under pseudogroup transformations of $\mathfrak{G}_{\tau}$, det $\mathbf{h}$ transforms as $\operatorname{det} \mathbf{h} \mapsto(\operatorname{det} \mathbf{h}) /$ $\left(\operatorname{det} \alpha_{j}^{i}\right)^{2}$, with $\left(\alpha_{j}^{i}\right) \in \operatorname{GL}(2, \mathbb{R})$. Therefore, the 1 -form $\sigma$ is $\mathfrak{G}_{\tau}^{(1)}$-invariant. The invariance of $\chi$ follows from the transformation rule

$$
\left(d h_{11} d h_{22}-d h_{12} d h_{12}\right) \mapsto\left(d h_{11} d h_{22}-d h_{12} d h_{12}\right) /\left(\operatorname{det} \alpha_{j}^{i}\right)^{2}
$$

under the pseudogroup transformations of $\mathfrak{G}_{\tau}$.

Consequently, the Cosgrove form

$$
\gamma=\chi-\frac{1}{4} \rho
$$

first introduced in [10, Eq. (2.3)], is also invariant.

An easy construction of the first-order scalar differential invariants follows from the consideration of the determinant $Q_{\mu}$ and the trace $C_{\mu}$ of the self-adjoint $(1,1)$-tensor field related to a symmetric bilinear form $\mu$ on $\mathcal{S}$, such as $\mu=\rho, \chi, \gamma$. In coordinates, if $\mu=\mu_{i j} d t^{i} d t^{j}$, then the corresponding $(1,1)$-tensor field has the components $\mu_{i}^{j}=\mu_{i s} \tilde{\mathbf{g}}^{s j}$, and

$$
Q_{\mu}=\frac{\operatorname{det} \mu_{i j}}{\operatorname{det} \tilde{\mathbf{g}}}, \quad C_{\mu}=\mu_{i j} \tilde{g}^{i j} .
$$

Choosing $\mu=\rho, \chi, \gamma$, we get four independent invariants $C_{\rho}, C_{\chi}, Q_{\chi}, Q_{\gamma}$, whereas $Q_{\rho}=$ 0 and $C_{\gamma}=C_{\chi}-\frac{1}{4} C_{\rho}$. A geometric meaning of $C_{\chi}$ is given in Proposition 10.

In coordinates, we have

1. $C_{\rho}=\frac{1}{(\operatorname{det} \mathbf{h})^{2}}(\operatorname{det} \mathbf{h})_{, i}(\operatorname{det} \mathbf{h})_{, j} \tilde{g}^{i j}$;

2. $C_{\chi}=\frac{1}{(\operatorname{det} \mathbf{h})} \tilde{g}^{i j}\left|\begin{array}{ll}h_{11, i} & h_{12, j} \\ h_{12, i} & h_{22, j}\end{array}\right|$;

3. $Q_{\chi}=\frac{\operatorname{det} \chi_{i j}}{\operatorname{det} \tilde{\mathbf{g}}}, \quad \chi_{i j}=\frac{1}{2 \operatorname{det} \mathbf{h}}\left|\begin{array}{ll}h_{11, i} & h_{12, j} \\ h_{21, i} & h_{22, j}\end{array}\right|+\frac{1}{2 \operatorname{det} \mathbf{h}}\left|\begin{array}{ll}h_{11, j} & h_{12, i} \\ h_{21, j} & h_{22, i}\end{array}\right|$;

4. $Q_{\gamma}=\frac{\operatorname{det} \gamma_{i j}}{\operatorname{det} \tilde{\mathbf{g}}}=\frac{1}{4(\operatorname{det} \mathbf{h})^{3} \operatorname{det} \tilde{\mathbf{g}}}\left|\begin{array}{ccc}h_{11} & h_{12} & h_{22} \\ h_{11,1} & h_{12,1} & h_{22,1} \\ h_{11,2} & h_{12,2} & h_{22,2}\end{array}\right|^{2}$.

Comma denotes partial differentiation.

Proposition 6 The functions $C_{\rho}, C_{\chi}, Q_{\chi}$ and $Q_{\gamma}$ are functionally independent first-order differential invariants in generic points of the jet space.

Proof Invariance follows from Lemma 1. Functional independence follows from the fact that the rank of the Jacobian at a generic point of the jet space is equal to 4. Obviously, the last condition is easily checked by computing the rank of a numeric matrix. 
Moreover, we notice that the 1 -form $\sigma$ is defined on $J^{1} \tau$ and horizontal with respect to $\pi_{1}:=\tau_{1} \circ \pi$, where $\tau_{1}: J^{1} \tau \rightarrow \mathcal{M}$. Thus, following [25], we consider the $\pi_{1}$-relative vector fields $\mathcal{X}$ and $\mathcal{X}^{\perp}$ on $\mathcal{S}$ such that $\sigma=\tilde{\mathbf{g}}(\mathcal{X},-)$ and $\left.\sigma=\mathcal{X}^{\perp}\right\lrcorner \operatorname{vol}_{\tilde{\mathbf{g}}}$, respectively, where

$$
\operatorname{vol}_{\tilde{\mathbf{g}}}=\sqrt{|\operatorname{det} \tilde{\mathbf{g}}|} d t^{1} \wedge d t^{2}
$$

is the volume form on $(\mathcal{S}, \tilde{\mathbf{g}})$.

Lemma 2 Under the pseudogroup action, for any metric $\mathbf{g}$ of the form (10), the vector field

$$
\mathcal{X}=\tilde{\mathbf{g}}^{i s} \frac{(\operatorname{det} \mathbf{h})_{s}}{\operatorname{det} \mathbf{h}} \partial_{t^{i}},
$$

is invariant, whereas the vector field

$$
\mathcal{X}^{\perp}=\frac{(\operatorname{det} \mathbf{h})_{, 2}}{(\operatorname{det} \mathbf{h}) \sqrt{|\operatorname{det} \tilde{\mathbf{g}}|}} \partial_{t^{1}}-\frac{(\operatorname{det} \mathbf{h})_{, 1}}{(\operatorname{det} \mathbf{h}) \sqrt{|\operatorname{det} \tilde{\mathbf{g}}|}} \partial_{t^{2}}
$$

transforms as $\mathcal{X}^{\perp} \mapsto \operatorname{sgn}\left(J_{\phi}\right) \mathcal{X}^{\perp}$. Moreover

$$
\tilde{\mathbf{g}}(\mathcal{X}, \mathcal{X})=C_{\rho}, \quad \tilde{\mathbf{g}}\left(\mathcal{X}, \mathcal{X}^{\perp}\right)=0, \quad \tilde{\mathbf{g}}\left(\mathcal{X}^{\perp}, \mathcal{X}^{\perp}\right)= \pm_{\tilde{\mathbf{g}}} C_{\rho} .
$$

where $\pm_{\tilde{\mathbf{g}}}=\operatorname{sgn} \operatorname{det} \tilde{\mathbf{g}}$. Hence, $\left\{\mathcal{X}, \mathcal{X}^{\perp}\right\}$ is a semi-invariant orthogonal frame on $\mathcal{S}$, when $C_{\rho} \neq 0$.

Proof In view of the invariance of $\sigma$ and the fact that $\operatorname{vol}_{\tilde{g}}$ is invariant only up to a sign, $\mathcal{X}$ and $\mathcal{X}^{\perp}$ have the specified invariance properties. Formulas (16) are routinely checked in adapted coordinates.

\subsection{The semi-invariant vector field $\mathcal{C}$ and scalar invariant $\ell_{\mathcal{C}}$}

The mapping $\pi: \mathcal{M} \rightarrow \mathcal{S}$ is a Riemannian submersion [5,9.12], with respect to metrics $\mathbf{g}$ and $\tilde{\mathbf{g}}$. Relative to this submersion, $\Xi$ will be referred to as the vertical distribution, whereas $\Xi^{\perp}$ as the horizontal distribution. Moreover, due to non-degeneracy condition (ii), the tangent bundle to $\mathcal{M}$ decomposes as $T \mathcal{M}=\Xi \oplus \Xi^{\perp}$, with $\Xi^{\perp}$ generated by the vector fields

$$
\mathbf{e}_{j}=\frac{\partial}{\partial t^{j}}-f_{j}^{k} \frac{\partial}{\partial z^{k}}, \quad j=1,2 .
$$

Recall that $f_{j}^{k}=f_{j s} h^{s k}$.

In view of this decomposition, one has the natural projections ver $=\operatorname{pr}_{\Xi}: T M \rightarrow \Xi$ and hor $=\operatorname{pr}_{\Xi}{ }^{\perp}: T M \rightarrow \Xi^{\perp}$ such that

$$
\operatorname{ver}\left(\frac{\partial}{\partial t^{j}}\right)=\operatorname{ver}\left(\frac{\partial}{\partial t^{j}}-f_{j}^{k} \frac{\partial}{\partial z^{k}}+f_{j}^{k} \frac{\partial}{\partial z^{k}}\right)=f_{j}^{k} \frac{\partial}{\partial z^{k}}, \quad \operatorname{ver}\left(\frac{\partial}{\partial z^{k}}\right)=\frac{\partial}{\partial z^{k}},
$$

and

$$
\operatorname{hor}\left(\frac{\partial}{\partial t^{j}}\right)=\operatorname{hor}\left(\frac{\partial}{\partial t^{j}}-f_{j}^{k} \frac{\partial}{\partial z^{k}}+f_{j}^{k} \frac{\partial}{\partial z^{k}}\right)=\frac{\partial}{\partial t^{j}}-f_{j}^{k} \frac{\partial}{\partial z^{k}}, \quad \operatorname{hor}\left(\frac{\partial}{\partial z^{k}}\right)=0 .
$$

In the adapted coordinates, the non-vanishing components of ver and hor are

$$
\operatorname{ver}_{j}^{k^{*}}=f_{j}^{k}, \quad \operatorname{ver}_{j^{*}}^{k^{*}}=\delta_{j}^{k}, \quad j, k=1,2,
$$

and

$$
\operatorname{hor}_{j}^{k}=\delta_{j}^{k}, \quad \operatorname{hor}_{j}^{k^{*}}=-f_{j}^{k}, \quad j, k=1,2 \text {, }
$$


respectively, where we use the notation $k^{*}=k+2$.

Remark 2 Every (relative) vector field $X$ on $\mathcal{S}$ can be uniquely lifted to an horizontal (relative) vector field $\widehat{X}$ on $\mathcal{M}$ which is $\pi$-related to $X$. In particular every invariant (relative) vector field on $\mathcal{S}$ can be uniquely lifted to an invariant (relative) vector field on $\mathcal{M}$. Moreover, the lift preserves the scalar product. In coordinates,

$$
\frac{\widehat{\partial}}{\partial t^{i}}=\frac{\partial}{\partial t^{i}}-f_{i}^{k} \frac{\partial}{\partial z^{k}}=\mathbf{e}_{i}, \quad i=1,2 .
$$

The geometry of the Riemannian submersion $\pi: \mathcal{M} \rightarrow \mathcal{S}$ can be described by using the Ehresmann curvature and the O'Neill tensors, which are naturally defined in terms of ver and hor.

The Ehresmann curvature is the tensor $\mathbf{c}: \mathcal{D}(\mathcal{M}) \otimes \mathcal{D}(\mathcal{M}) \rightarrow \mathcal{D}(\mathcal{M})$ defined in terms of the Lie bracket by

$$
\mathbf{c}\left(W_{1}, W_{2}\right)=\operatorname{ver}\left[\text { hor } W_{1} \text {, hor } W_{2}\right],
$$

for any two vector fields $W_{1}, W_{2} \in \mathcal{D}(\mathcal{M})$. This is an antisymmetric tensor whose nonzero components in adapted coordinates are

$$
\mathbf{c}_{i j}^{k^{*}}=\partial_{j} f_{i}^{k}-\partial_{i} f_{j}^{k},
$$

where $k^{*}=k+2$. It is easily checked that $\mathbf{c}$ is traceless, $\mathbf{c}_{a b}^{a}=0$. Of course, $\mathbf{c}=0$ if and only if $\Xi^{\perp}$ is involutive.

The curvature vector field $\mathcal{C}$ is defined as

$$
\mathcal{C}=\frac{\mathbf{c}\left(\partial_{t_{1}}, \partial_{t_{2}}\right)}{\sqrt{|\operatorname{det} \tilde{\mathbf{g}}|}}
$$

This is a semi-invariant vector field, since it transforms as $\mathcal{C} \mapsto\left(\operatorname{sgn} J_{\phi}\right) \mathcal{C}$ under pseudogroup transformations (2). Indeed, the numerator and denominator of (19) transform as $\mathbf{c}\left(\partial_{t_{1}}, \partial_{t_{2}}\right)=$ $\operatorname{ver}\left[\operatorname{hor} \partial_{t_{1}}\right.$, hor $\left.\partial_{t_{2}}\right] \mapsto \operatorname{ver}\left[\operatorname{hor} \partial_{t_{1}}\right.$, hor $\left.\partial_{t_{2}}\right] / J_{\phi}$ and $\sqrt{|\operatorname{det} \tilde{\mathbf{g}}|} \mapsto \sqrt{|\operatorname{det} \tilde{\mathbf{g}}|} /\left|J_{\phi}\right|$, respectively. In coordinates,

$$
\mathcal{C}=\mathcal{C}^{k} \frac{\partial}{\partial z^{k}}, \quad \mathcal{C}^{k}=\frac{\partial_{t^{2}} f_{1}^{k}-\partial_{t^{1}} f_{2}^{k}}{\sqrt{|\operatorname{det} \tilde{\mathbf{g}}|}}, \quad k=1,2 .
$$

Consider now the scalar invariant $\ell_{\mathcal{C}}=\mathbf{g}(\mathcal{C}, \mathcal{C})$, i.e., the squared length of $\mathcal{C}$. Obviously from the coordinate formulas, $\ell_{\mathcal{C}}$ is given by

$$
\ell_{\mathcal{C}}=\mathbf{g}(\mathcal{C}, \mathcal{C})=h_{k l} \mathcal{C}^{k} \mathcal{C}^{l}=\frac{h_{k l}\left(\partial_{t^{2}} f_{1}^{k}-\partial_{t^{1}} f_{2}^{k}\right)\left(\partial_{t^{2}} f_{1}^{l}-\partial_{t^{1}} f_{2}^{l}\right)}{|\operatorname{det} \tilde{\mathbf{g}}|}
$$

In the generic case, $\ell_{\mathcal{C}}$ is functionally independent from the previous four invariants $C_{\rho}, C_{\chi}, C_{\gamma}, Q_{\chi}$. Consequently, $\ell_{\mathcal{C}}$ is the fifth scalar invariant sought. Summarizing, we have the following lemma.

Lemma 3 For any metric $\mathbf{g}$ of the form (10), the curvature vector field $\mathcal{C}$ transforms as $\mathcal{C} \mapsto\left(\operatorname{sgn} J_{\phi}\right) \mathcal{C}$ under the pseudogroup action $(2)$. Therefore, $\ell_{\mathcal{C}}=\mathbf{g}(\mathcal{C}, \mathcal{C})$ is a scalar differential invariant.

We say that a metric belongs to the Kundu class when $\ell_{\mathcal{C}} \equiv 0$, i.e., when $\mathcal{C}$ is null. Vacuum Einstein metrics in this class have been studied by Kundu [21]. 


\section{3 $0^{\prime}$ Neill tensors $A$ and T. The invariant and semi-invariant vector fields $\mathcal{H}$ and $\mathcal{H}^{\perp}$ in the case when $C_{\rho} \neq 0$}

To construct further invariants, we introduce also a semi-invariant orthogonal frame on $\Xi^{\perp}$ by employing the O'Neill tensors $\mathbf{A}$ and $\mathbf{T}[5,28]$. These tensors are defined by

$$
\begin{aligned}
& \mathbf{A}\left(W_{1}, W_{2}\right)=\mathbf{O}\left(W_{1}, W_{2}\right)+\mathbf{E}\left(W_{1}, W_{2}\right), \\
& \mathbf{T}\left(W_{1}, W_{2}\right)=\mathbf{N}\left(W_{1}, W_{2}\right)+\mathbf{L}\left(W_{1}, W_{2}\right),
\end{aligned}
$$

where

$$
\begin{array}{ll}
\mathbf{O}\left(W_{1}, W_{2}\right)=\operatorname{ver}\left(\nabla_{\text {hor } W_{1}} \text { hor } W_{2}\right), & \mathbf{E}\left(W_{1}, W_{2}\right)=\operatorname{hor}\left(\nabla_{\text {hor } W_{1}} \operatorname{ver} W_{2}\right), \\
\mathbf{N}\left(W_{1}, W_{2}\right)=\operatorname{ver}\left(\nabla_{\text {ver } W_{1}} \text { hor } W_{2}\right), & \mathbf{L}\left(W_{1}, W_{2}\right)=\operatorname{hor}\left(\nabla_{\text {ver } W_{1}} \operatorname{ver} W_{2}\right),
\end{array}
$$

for arbitrary vector fields $W_{1}, W_{2}$ on $\mathcal{M}$.

As is well known, see [5, §9.24],

$$
\mathbf{A}\left(\text { hor } W_{1} \text {, hor } W_{2}\right)=\frac{1}{2} \mathbf{c}\left(W_{1}, W_{2}\right),
$$

while $\mathbf{A}\left(\right.$ hor $W_{1}$, hor $\left.W_{2}\right)=\mathbf{A}\left(W_{1}\right.$, hor $\left.W_{2}\right)=\mathbf{O}\left(W_{1}, W_{2}\right)$, hence

$$
\mathbf{O}\left(W_{1}, W_{2}\right)=\mathbf{A}\left(W_{1} \text {, hor } W_{2}\right)=\frac{1}{2} \mathbf{c}\left(W_{1}, W_{2}\right)
$$

meaning that in adapted coordinates components of $\mathbf{O}$ are simply one half of those of $\mathbf{c}$ given by formulas (18).

Remark 3 The second fundamental form of the fibers of the submersion is defined by $\left.\overline{\mathbf{T}}\right|_{\Xi}$, the restriction of $T$ to $\Xi$. Hence, $\left.\overline{\mathbf{T}}\right|_{\Xi}=0$ if and only if the Riemannian submersion has totally geodesic fibers. Moreover, $\Xi^{\perp}$ is completely integrable iff the restriction of $\mathbf{A}$ to $\Xi^{\perp}$ identically vanishes. In particular, when $\mathbf{A}=0$, then $\Xi^{\perp}$ is completely integrable.

To construct a semi-invariant orthogonal frame in $\Xi^{\perp}$, we consider the mean curvature vector field $\mathcal{H}$, defined as

$$
\mathcal{H}=\sum_{s=1}^{2} \mathbf{T}\left(\mathbf{v}_{s}, \mathbf{v}_{s}\right),
$$

for any vertical orthonormal frame $\left\{\mathbf{v}_{1}, \mathbf{v}_{2}\right\}$. Obviously, $\mathcal{H}$ is invariant with respect to the action of $\mathfrak{G}_{\tau}^{(1)}$. In adapted coordinates, $\mathcal{H}$ is the contraction $\mathcal{H}^{a}=\mathbf{g}^{k l} \mathbf{T}_{k l}^{a}$. Hence,

$$
\mathcal{H}=\mathcal{H}^{i}\left(\frac{\partial}{\partial t^{i}}-f_{i}^{k} \frac{\partial}{\partial z^{k}}\right)
$$

where

$$
\mathcal{H}^{i}=-\frac{1}{2} \tilde{\mathbf{g}}^{i s} \frac{(\operatorname{det} \mathbf{h})_{s}}{\operatorname{det} \mathbf{h}}, \quad i=1,2 .
$$

By comparing (14) and (21), one sees that $\mathcal{H} \in \Xi^{\perp}$ is a lifted vector field; more precisely, $\mathcal{H}=-\frac{1}{2} \hat{\mathcal{X}}$. The squared length of $\mathcal{H}$ is easily seen to be $\ell_{\mathcal{H}}=\mathbf{g}(\mathcal{H}, \mathcal{H})=\frac{1}{4} C_{\rho}$.

In the case when $C_{\rho} \neq 0$, to complete the sought semi-invariant orthogonal frame, we introduce the orthogonal complement $\mathcal{H}^{\perp} \in \Xi^{\perp}$ by lifting the vector field $-\frac{1}{2} \mathcal{X}^{\perp}$, see formula (15). Then, since the lift preserves the scalar product, one has $\mathbf{g}\left(\mathcal{H}, \mathcal{H}^{\perp}\right)=0$ and

$$
\ell_{\mathcal{H}^{\perp}}=\mathbf{g}\left(\mathcal{H}^{\perp}, \mathcal{H}^{\perp}\right)=\mathbf{g}\left(-\frac{1}{2} \mathcal{X}^{\perp},-\frac{1}{2} \mathcal{X}^{\perp}\right)= \pm_{\tilde{\mathbf{g}}} \frac{1}{4} C_{\rho}= \pm_{\tilde{\mathbf{g}}} \ell_{\mathcal{H}}
$$


In coordinates,

$$
\mathcal{H}^{\perp}=\left(\mathcal{H}^{\perp}\right)^{i}\left(\frac{\partial}{\partial t^{i}}-f_{i}^{k} \frac{\partial}{\partial z^{k}}\right)
$$

where

$$
\left(\mathcal{H}^{\perp}\right)^{1}=-\frac{1}{2} \frac{(\operatorname{det} \mathbf{h})_{, 2}}{(\operatorname{det} \mathbf{h}) \sqrt{|\operatorname{det} \tilde{\mathbf{g}}|}}, \quad\left(\mathcal{H}^{\perp}\right)^{2}=\frac{1}{2} \frac{(\operatorname{det} \mathbf{h})_{, 1}}{(\operatorname{det} \mathbf{h}) \sqrt{|\operatorname{det} \tilde{\mathbf{g}}|}} .
$$

The pair $\mathcal{H}, \mathcal{H}^{\perp}$ is the sought semi-invariant orthogonal frame in $\Xi^{\perp}$ when $C_{\rho} \neq 0$.

\subsection{The semi-invariant orthogonal frame $\left\{\mathcal{H}, \mathcal{H}^{\perp}, \mathcal{C}, \mathcal{C}^{\perp}\right\}$ in the case when $c_{\rho} \ell_{\mathcal{C}} \neq 0$}

By construction, $\mathcal{C} \in \Xi$, where $\Xi$ is two-dimensional. Let $\mathcal{C}^{\perp}$ be the orthogonal complement of $\mathcal{C}$ in $\Xi$, uniquely determined by the requirements $\mathbf{g}\left(\mathcal{C}, \mathcal{C}^{\perp}\right)=0, \operatorname{vol}_{\mathbf{h}}\left(\mathcal{C}, \mathcal{C}^{\perp}\right)>0, \ell_{\mathcal{C}^{\perp}}=$ $\mathbf{g}\left(\mathcal{C}^{\perp}, \mathcal{C}^{\perp}\right)= \pm_{\mathbf{h}} \ell_{\mathcal{C}}$, where $\pm_{\mathbf{h}}=\operatorname{sgn} \operatorname{det} \mathbf{h}$, and

$$
\operatorname{vol}_{\mathbf{h}}=\sqrt{|\operatorname{det} \mathbf{h}|} d z^{1} \wedge d z^{2}
$$

is the $\left(t^{1}, t^{2}\right)$-dependent volume form of the orbits with metric $\mathbf{h}=h_{i j} d z^{i} d z^{j}$. In coordinates,

$$
\mathcal{C}^{\perp}=\mathcal{C}^{\perp k} \frac{\partial}{\partial z^{k}}, \quad \mathcal{C}^{\perp 1}=\frac{h_{s 2} \mathcal{C}^{s}}{\sqrt{|\operatorname{det} \mathbf{h}|}}, \quad \mathcal{C}^{\perp 2}=\frac{-h_{s 1} \mathcal{C}^{s}}{\sqrt{|\operatorname{det} \mathbf{h}|}} .
$$

The vector field $\mathcal{C}^{\perp}$ is semi-invariant, since it transforms as

$$
\mathcal{C}^{\perp} \mapsto\left(\operatorname{sgn} J_{\phi}\right)\left(\operatorname{sgn} \operatorname{det} \alpha_{j}^{i}\right) \mathcal{C}^{\perp}
$$

under pseudogroup transformations (2). Hence, when $\ell_{\mathcal{C}} \neq 0$, the pair $\mathcal{C}, \mathcal{C}^{\perp}$ defines a semiinvariant orthogonal frame on $\Xi$.

Summarizing, we have the following proposition about the semi-invariant frame $\left\{\mathcal{H}, \mathcal{H}^{\perp}\right.$, $\left.\mathcal{C}, \mathcal{C}^{\perp}\right\}$

Proposition 7 In the case when $C_{\rho} \ell_{\mathcal{C}} \neq 0$, the pairs of vector fields $\mathcal{H}, \mathcal{H}^{\perp} \in \Xi^{\perp}$ and $\mathcal{C}, \mathcal{C}^{\perp} \in \Xi$ form a semi-invariant orthogonal frame on $\mathcal{M}$. In particular, under the pseudogroup action (2), these fields transform as

$$
\begin{aligned}
& \mathcal{H} \mapsto \mathcal{H}, \quad \mathcal{H}^{\perp} \mapsto\left(\operatorname{sgn} J_{\phi}\right) \mathcal{H}^{\perp}, \\
& \mathcal{C} \mapsto\left(\operatorname{sgn} J_{\phi}\right) \mathcal{C}, \quad \mathcal{C}^{\perp} \mapsto\left(\operatorname{sgn} J_{\phi}\right)\left(\operatorname{sgn} \operatorname{det} \alpha_{j}^{i}\right) \mathcal{C}^{\perp}
\end{aligned}
$$

Moreover, the nonzero components of $\mathbf{g}$ in this frame are the invariants

$$
\begin{aligned}
\mathbf{g}(\mathcal{H}, \mathcal{H}) & =\ell_{\mathcal{H}}=\frac{1}{4} C_{\rho}, \quad \mathbf{g}\left(\mathcal{H}^{\perp}, \mathcal{H}^{\perp}\right)=\ell_{\mathcal{H}^{\perp}}= \pm_{\tilde{\mathbf{g}}} \frac{1}{4} C_{\rho}, \\
\mathbf{g}(\mathcal{C}, \mathcal{C}) & =\ell_{\mathcal{C}}, \quad \mathbf{g}\left(\mathcal{C}^{\perp}, \mathcal{C}^{\perp}\right)=\ell_{\mathcal{C}^{\perp}}= \pm_{\mathbf{h}} \ell_{\mathcal{C}}
\end{aligned}
$$

where $\pm_{\tilde{\mathbf{g}}}=\operatorname{sgn} \operatorname{det} \tilde{\mathbf{g}}, \pm_{\mathbf{h}}=\operatorname{sgn} \operatorname{det} \mathbf{h}$. 


\subsection{Further first-order scalar invariants}

In this section we discover three new semi-invariants $\Theta_{\mathrm{I}}, \Theta_{\mathrm{II}}, \Theta_{\mathrm{III}}$ by examining the components of the $\mathrm{O}^{\prime}$ Neill tensors in the frame $\left\{\mathcal{H}, \mathcal{H}^{\perp}, \mathcal{C}, \mathcal{C}^{\perp}\right\}$. This frame is well defined only when $C_{\rho} \ell_{\mathcal{C}} \neq 0$.

In the rest of the paper, the components of a tensor $\mathbf{W}$ with respect to the frame $\left\{\mathcal{H}, \mathcal{H}^{\perp}, \mathcal{C}, \mathcal{C}^{\perp}\right\}$ will be denoted by $\mathbf{W}_{(c)(d) \ldots}^{(a)(b) \ldots}$. Thus, e.g.

$$
\mathbf{g}_{(1)(1)}=\mathbf{g}(\mathcal{H}, \mathcal{H}), \quad \mathbf{g}_{(1)(2)}=\mathbf{g}\left(\mathcal{H}, \mathcal{H}^{\perp}\right),
$$

etc. Now, in view of Proposition 7 the nonzero components $\mathbf{g}_{(a)(b)}$ are scalar invariants, which coincide up to a sign with $\ell_{\mathcal{H}}$ and $\ell_{\mathcal{C}}$. Analogously, the only nonzero components $\mathbf{A}_{(b)(c)}^{(a)}$ of the O'Neill tensor $\mathbf{A}$ are

$$
\mathbf{A}_{(2)(3)}^{(1)}=-\frac{1}{2} \ell_{\mathcal{C}}, \quad \mathbf{A}_{(1)(3)}^{(2)}= \pm_{\tilde{\mathbf{g}}} \frac{1}{2} \ell_{\mathcal{C}}, \quad \mathbf{A}_{(1)(2)}^{(3)}=-\frac{1}{2} \ell_{\mathcal{H}}, \quad \mathbf{A}_{(2)(1)}^{(3)}=\frac{1}{2} \ell_{\mathcal{H}},
$$

yielding no new scalar invariant.

On the contrary, the nonzero components $\mathbf{T}_{(b)(c)}^{(a)}$ of the O'Neill tensor $\mathbf{T}$ are much more interesting. Indeed, in order of increasing complexity, the nonzero components of $\mathbf{T}$ are

$$
\mathbf{T}_{(3)(2)}^{(3)}, \mathbf{T}_{(4)(2)}^{(3)}, \mathbf{T}_{(4)(1)}^{(3)}, \mathbf{T}_{(3)(1)}^{(3)}, \mathbf{T}_{(4)(1)}^{(4)} .
$$

In view of (23), the first three components of this quintuple are semi-invariants, whereas $\mathbf{T}_{(3)(1)}^{(3)}$ and $\mathbf{T}_{(4)(1)}^{(4)}$ are invariants. Before exploring them in detail, we also introduce two semi-invariant tensors of type $(1,1)$ defined by

$$
\mathbf{T}_{\mathcal{C}}(U)=\mathbf{T}(\mathcal{C}, U), \quad \mathbf{T}_{\mathcal{C}^{\perp}}(U)=\mathbf{T}\left(\mathcal{C}^{\perp}, U\right)
$$

for an arbitrary vector field $U$ on $\mathcal{M}$. Thus,

$$
\left(\mathbf{T}_{\mathcal{C}}\right)_{j}^{k}=\mathbf{T}_{s j}^{k} \mathcal{C}^{s}, \quad\left(\mathbf{T}_{\mathcal{C}^{\perp}}\right)_{j}^{k}=\mathbf{T}_{s j}^{k} \mathcal{C}^{\perp s}
$$

Of interest are

$$
\Theta_{\mathcal{C}}=\operatorname{det} \mathbf{T}_{\mathcal{C}}, \quad \Theta_{\mathcal{C}^{\perp}}=\operatorname{det} \mathbf{T}_{\mathcal{C}^{\perp}}
$$

since they are invariants, while the traces $\left(\mathbf{T}_{\mathcal{C}}\right)_{i}^{i}=\left(\mathbf{T}_{\mathcal{C}^{\perp}}\right)_{i}^{i}=0$ vanish. We could choose $\Theta_{\mathcal{C}}$ or $\Theta_{\mathcal{C}^{\perp}}$ as the missing sixth invariant, but there exist semi-invariants of lower complexity. To find these semi-invariants, we first consider the vector fields

$$
\mathcal{T}=\mathbf{T}_{(3)(2)}=\mathbf{T}\left(\mathcal{C}, \mathcal{H}^{\perp}\right), \quad \mathcal{T}^{\perp}=\mathbf{T}_{(4)(2)}=\mathbf{T}\left(\mathcal{C}^{\perp}, \mathcal{H}^{\perp}\right) .
$$

They are, respectively, invariant and semi-invariant and constitute another orthogonal frame in $\Xi$. Moreover, denoting by

$$
\ell_{\mathcal{T}}=\mathbf{g}(\mathcal{T}, \mathcal{T}), \quad \ell_{\mathcal{T}^{\perp}}=\mathbf{g}\left(\mathcal{T}^{\perp}, \mathcal{T}^{\perp}\right)
$$

the squared lengths, which are invariants, we have $\ell_{\mathcal{T} \perp}= \pm_{\mathbf{h}} \ell_{\mathcal{T}}$. The angles between $\mathcal{T}$ or $\mathcal{T}^{\perp}$ and $\mathcal{C}$ or $\mathcal{C}^{\perp}$ (they all lie in $\Xi$ ) are scalar semi-invariants, proportional to $\mathbf{g}(\mathcal{T}, \mathcal{C})=\ell_{\mathcal{C}} \mathbf{T}_{(3)(2)}^{(3)}$ and $\mathbf{g}\left(\mathcal{T}^{\perp}, \mathcal{C}\right)=\ell_{\mathcal{C}} \mathbf{T}_{(4)(2)}^{(3)}$. 
The coordinate description of the invariants and semi-invariants introduced above involves the three relatively simple semi-invariants

$$
\begin{aligned}
& \Theta_{\mathrm{I}}=\frac{1}{|\operatorname{det} \mathbf{h}|^{1 / 2}|\operatorname{det} \tilde{\mathbf{g}}|^{1 / 2}}\left|\begin{array}{ccc}
h_{11,1} & h_{12,1} & h_{22,1} \\
h_{11,2} & h_{12,2} & h_{22,2} \\
\left(\mathcal{C}^{2}\right)^{2} & -\mathcal{C}^{1} \mathcal{C}^{2} & \left(\mathcal{C}^{1}\right)^{2}
\end{array}\right|, \\
& \Theta_{\mathrm{II}}=4 \mathbf{g}(\mathcal{T}, \mathcal{C})=\frac{1}{\operatorname{det} \mathbf{h}|\operatorname{det} \tilde{\mathbf{g}}|^{1 / 2}}\left|\begin{array}{ccc}
h_{11,1} & h_{12,1} & h_{22,1} \\
h_{11,2} & h_{12,2} & h_{22,2} \\
-2 h_{1 k} \mathcal{C}^{k} \mathcal{C}^{2} & h_{11}\left(\mathcal{C}^{1}\right)^{2}-h_{22}\left(\mathcal{C}^{2}\right)^{2} & 2 h_{2 k} \mathcal{C}^{1} \mathcal{C}^{k}
\end{array}\right|,
\end{aligned}
$$

and

$$
\Theta_{\text {III }}=\frac{1}{|\operatorname{det} \mathbf{h}|^{3 / 2}|\operatorname{det} \tilde{\mathbf{g}}|^{1 / 2}}\left|\begin{array}{ccc}
h_{11,1} & h_{12,1} & h_{22,1} \\
h_{11,2} & h_{12,2} & h_{22,2} \\
\left(h_{1 k} \mathcal{C}^{k}\right)^{2} & h_{1 k} h_{2 l} \mathcal{C}^{k} \mathcal{C}^{l} & \left(h_{2 l} \mathcal{C}^{l}\right)^{2}
\end{array}\right|,
$$

which are such that

$$
\Theta_{\mathrm{I}}^{2}=16 \Theta_{\mathcal{C}}, \quad \Theta_{\mathrm{II}}=4 \ell_{\mathcal{C}} \mathbf{T}_{(3)(2)}^{(3)}, \quad \Theta_{\mathrm{III}}^{2}= \pm_{\tilde{\mathbf{g}} \mathbf{h}} 16 \Theta_{\mathcal{C}^{\perp}} .
$$

Summarizing,

$$
C_{\rho}, C_{\chi}, C_{\gamma}, Q_{\chi}, Q_{\gamma}, \Theta_{\mathcal{C}}, \Theta_{\mathcal{C}^{\perp}}, \ell_{\mathcal{C}}, \ell_{\mathcal{T}}, \mathbf{T}_{(3)(1)}^{(3)}, \mathbf{T}_{(4)(1)}^{(4)}
$$

and

$$
\Theta_{\mathrm{I}}, \Theta_{\mathrm{II}}, \Theta_{\mathrm{III}}, \mathbf{T}_{(3)(2)}^{(3)}, \mathbf{T}_{(4)(2)}^{(3)}, \mathbf{T}_{(4)(1)}^{(3)},
$$

are two (relatively simple) sets of first-order scalar invariants and semi-invariants, respectively.

As we already know, at most six first-order invariants can be functionally independent. Since the semi-invariants can only change their sign under the pseudogroup action (2),

$$
C_{\rho}, C_{\chi}, Q_{\chi}, Q_{\gamma}, \ell_{\mathcal{C}},\left(\Theta_{\mathrm{I}}\right)^{2}
$$

turn out to be the simplest six functionally independent scalar invariants.

Proposition 8 In the orthogonally intransitive case, the scalar differential invariants $I_{1}=$ $C_{\rho}, I_{2}=C_{\chi}, I_{3}=Q_{\chi}, I_{4}=Q_{\gamma}, I_{5}=\ell_{\mathcal{C}}$ and $I_{6}=\left(\Theta_{\mathrm{I}}\right)^{2}$ form a maximal system of functionally independent scalar differential invariants of the first order in generic points of the jet space.

Proof The rank of the Jacobian at a generic point of the jet space is equal to six.

Thus, all invariants can be expressed in terms of $C_{\rho}, C_{\chi}, Q_{\chi}, Q_{\gamma}, \ell_{\mathcal{C}},\left(\Theta_{\mathrm{I}}\right)^{2}$. The simplest functional relations are provided by (24) and

$$
\frac{\Theta_{\mathrm{II}}^{2}}{16 \ell_{\mathcal{C}}^{2}} \pm_{\mathbf{h}}\left(\mathbf{T}_{(4)(2)}^{(3)}\right)^{2} \pm_{\tilde{\mathbf{g}}} \frac{1}{4}\left(Q_{\chi}-Q_{\gamma}\right)=0 .
$$

Moreover, the relations among $Q_{\chi}, Q_{\gamma}, \ell_{\mathcal{C}}, \Theta_{\mathrm{I}}, \Theta_{\mathrm{II}}, \Theta_{\mathrm{III}}$ are

$$
-2 \ell_{\mathcal{C}} \sqrt{\left|Q_{\gamma}\right|}+\Theta_{\mathrm{I}}+\Theta_{\mathrm{III}}=0
$$

and

$$
\pm_{\tilde{\mathbf{g}}} 4 Q_{\chi} \ell_{\mathcal{C}}^{2} \mp_{\mathbf{h}} 8 \Theta_{\mathrm{I}} \sqrt{\left|Q_{\gamma}\right|} \ell_{\mathcal{C}} \pm_{\mathbf{h}} 4 \Theta_{\mathrm{I}}^{2}+\Theta_{\mathrm{II}}^{2}=0,
$$

where $Q_{\gamma}$ is the scalar invariant defined in Sect. 5.1. 


\section{Additional second-order invariants}

Besides the fourteen second-order Carminati-McLenaghan invariants [7] available for every four-dimensional metric, there are additional invariants originating in the submersion structure.

An infinite sequence of higher-order scalar differential invariants is obtained by repeatedly applying invariant or semi-invariant differentiations to the first-order scalar invariants listed in Proposition 8. Invariant differentiations correspond to vector fields on the orbit space $\mathcal{S}$. We already introduced two such vector fields $\mathcal{X}, \mathcal{X}^{\perp}$ in Sect. 5.1, assuming that $C_{\rho} \neq 0$; the corresponding invariant differentiations will be denoted $X, X^{\perp}$. In coordinates,

$$
\begin{aligned}
X & =\tilde{\mathbf{g}}^{i s} \frac{(\operatorname{det} \mathbf{h})_{, s}}{\operatorname{det} \mathbf{h}} D_{t^{i}}, \\
X^{\perp} & =\frac{(\operatorname{det} \mathbf{h})_{, 2}}{(\operatorname{det} \mathbf{h}) \sqrt{|\operatorname{det} \tilde{\mathbf{g}}|}} D_{t^{1}}-\frac{(\operatorname{det} \mathbf{h})_{, 1}}{(\operatorname{det} \mathbf{h}) \sqrt{|\operatorname{det} \tilde{\mathbf{g}}|}} D_{t^{2}},
\end{aligned}
$$

cf. Lemma 2. Therefore, according to Proposition 8, we have the 12 second-order invariants $X\left(I_{j}\right), X^{\perp}\left(I_{j}\right), j=1, \ldots, 6$.

A related construction of higher-order invariants is as follows. Let $\mathcal{I}^{1}, \mathcal{I}^{2}$ be two scalar invariants such that

$$
\Delta=\left|\begin{array}{cc}
X \mathcal{I}^{1} & X \mathcal{I}^{2} \\
X^{\perp} \mathcal{I}^{1} & X^{\perp} \mathcal{I}^{2}
\end{array}\right| \neq 0
$$

For any other scalar invariant $\phi$, define scalar invariants $\phi_{\mathcal{I}^{1}}, \phi_{\mathcal{I}^{2}}$ by

$$
\phi_{\mathcal{I}^{1}}=\frac{1}{\Delta}\left|\begin{array}{cc}
X \phi & X \mathcal{I}^{2} \\
X^{\perp} \phi & X^{\perp} \mathcal{I}^{2}
\end{array}\right|, \quad \phi_{\mathcal{I}^{2}}=\frac{1}{\Delta}\left|\begin{array}{cc}
X \mathcal{I}^{1} & X \phi \\
X^{\perp} \mathcal{I}^{1} & X^{\perp} \phi
\end{array}\right| .
$$

When $\mathcal{I}^{1}, \mathcal{I}^{2}$ are of the first order and $\phi$ is of order $n \geq 1$, then $\phi_{\mathcal{I}^{1}}, \phi_{\mathcal{I}^{2}}$ are, in general, of order $n+1$. The invariants $\phi_{\mathcal{I}^{1}}, \phi_{\mathcal{I}^{2}}$ have an obvious geometric meaning. The scalar invariants $\mathcal{I}^{1}, \mathcal{I}^{2}$ restricted to the orbit space $\mathcal{S}$ (see Remark 1) constitute a local coordinate system on $\mathcal{S}$ if they are functionally independent or, equivalently, when $\Delta \neq 0$ (still assuming that $C_{\rho} \neq 0$ ). Let $\phi$ be any other invariant restricted to $\mathcal{S}$. Solving $\left\{X \phi=X \mathcal{I}^{i} \partial \phi / \partial \mathcal{I}^{i}\right.$, $\left.X^{\perp} \phi=X^{\perp} \mathcal{I}^{i} \partial \phi / \partial \mathcal{I}^{i}\right\}$ as a linear system for $\partial \phi / \partial \mathcal{I}^{i}$, we see that the partial derivative $\partial \phi / \partial \mathcal{I}^{i}$ is equal to $\phi_{\mathcal{I}^{i}}$.

Additional invariants arise by means of formula (13) for suitable symmetric bilinear forms on the orbit space $\mathcal{S}$. For instance, denoting ric $=\operatorname{Ric}(\tilde{\mathbf{g}})$ the Ricci form of $\mathcal{S}$, one has the invariants $Q_{\text {ric }}$ and also $C_{\text {ric }}=\operatorname{Sc}_{\mathcal{S}}$, the scalar curvature of $\mathcal{S}$. Along the same line, in view of the invariance of $\sigma=d \ln |\operatorname{det} \mathbf{h}|$, the Hessian $v=H e s s(\ln |\operatorname{det} \mathbf{h}|)$, defined by

$$
\left.v(U, V)=\operatorname{Hess}(\ln |\operatorname{det} \mathbf{h}|)(U, V)=U\lrcorner \nabla_{V} d(\ln |\operatorname{det} \mathbf{h}|)=V\right\lrcorner \nabla_{U} d(\ln |\operatorname{det} \mathbf{h}|)
$$

for all vector fields $U, V$ on $\mathcal{S}$, is another symmetric bilinear form on $\mathcal{S}$. Hence, one obtains two additional invariants $Q_{v}$ and $C_{v}=\Delta_{\mathcal{S}} \ln |\operatorname{det} \mathbf{h}|$, where $\Delta_{\mathcal{S}}$ is the Laplace-Beltrami operator. It is worth mentioning here that

$$
C_{v}^{\prime}:=C_{v}-2 C_{\chi}+C_{\rho}=g^{i j} h^{k l} h_{k l, i j}+g_{i}^{i j} h^{k l} h_{k l, j}+\frac{1}{2} g^{i j} g^{m, n} g_{m n, i} h^{k l} h_{k l, j}
$$

has a noteworthy simpler coordinate expression than $C_{v}$ itself.

Proposition 9 In the orthogonally intransitive case, the invariants $I_{j}, X\left(I_{j}\right), X^{\perp}\left(I_{j}\right), j=$ $1, \ldots, 6$ (see Proposition 8), $C_{\text {ric }}$ and $C_{v}\left(\right.$ or $\left.C_{v}^{\prime}\right)$ form a maximal system of 20 generically 
functionally independent scalar differential invariants of order less than 3. All other secondorder invariants are functionally dependent on these.

Proof The rank of the Jacobian at a generic point of the jet space is equal to 20 .

For example, one can check that

$$
\begin{aligned}
& Q_{\text {ric }}=\frac{1}{4}\left(C_{\text {ric }}\right)^{2}, \\
& \quad 4 C_{\rho}^{2} Q_{\nu}+\left(X C_{\rho}\right)^{2} \pm_{\tilde{\mathbf{g}}}\left(X^{\perp} C_{\rho}\right)^{2}-2 C_{\nu} C_{\rho} X C_{\rho}=0 .
\end{aligned}
$$

If $C_{\rho} \neq 0$, then the last formula allows us to express $Q_{v}$ in terms of $C_{\rho}, X C_{\rho}, X^{\perp} C_{\rho}$ and $C_{\nu}$.

To extend the set of geometrically meaningful invariants, we consider the sectional curvatures

$$
K(\Xi)=\frac{g\left(R\left(\partial_{z^{1}}, \partial_{z^{2}}\right) \partial_{z^{1}}, \partial_{z^{2}}\right)}{g\left(\partial_{z^{1}}, \partial_{z^{1}}\right) g\left(\partial_{z^{2}}, \partial_{z^{2}}\right)-g\left(\partial_{z^{1}}, \partial_{z^{2}}\right)^{2}}
$$

and

$$
K\left(\Xi^{\perp}\right)=\frac{g\left(R\left(\mathbf{e}_{1}, \mathbf{e}_{2}\right) \mathbf{e}_{1}, \mathbf{e}_{2}\right)}{g\left(\mathbf{e}_{1}, \mathbf{e}_{1}\right) g\left(\mathbf{e}_{2}, \mathbf{e}_{2}\right)-g\left(\mathbf{e}_{1}, \mathbf{e}_{2}\right)^{2}}
$$

of $\Xi$ and $\Xi^{\perp}$, respectively, with vectors $\mathbf{e}_{i}$ being given by formulas (17).

Proposition 10 We have

$$
K(\Xi)=-\frac{1}{4} C_{\chi}, \quad K\left(\Xi^{\perp}\right)=\frac{1}{2} C_{\text {ric }} \mp \tilde{\mathbf{g}} \frac{3}{4} \ell_{\mathcal{C}} .
$$

Proof Both formulas are routinely checked in adapted coordinates.

Finally, second-order invariants can be also obtained from the commutator $\left[\mathcal{X}, \mathcal{X}^{\perp}\right]$, which lies in $\Xi$, hence is a linear combination of $\mathcal{X}$ and $\mathcal{X}^{\perp}$. However, the coefficients are rather simple expressions in $C_{\rho}, X C_{\rho}, X^{\perp} C_{\rho}$ and $C_{\nu}$.

Proposition 11 Let $C_{\rho} \neq 0$. Then, the (semi-)invariant differentiations $\mathcal{X}$ and $\mathcal{X}^{\perp}$ satisfy the commutation relations

$$
\left[\mathcal{X}, \mathcal{X}^{\perp}\right]=J_{1} \mathcal{X}+J_{2} \mathcal{X}^{\perp}
$$

where

$$
J_{1}=-\frac{X^{\perp} C_{\rho}}{C_{\rho}}, \quad J_{2}=\frac{X C_{\rho}}{C_{\rho}}-C_{\nu} .
$$

Proof By orthogonality, we have

$$
J_{1}=\frac{\tilde{\mathbf{g}}\left(\mathcal{X},\left[\mathcal{X}, \mathcal{X}^{\perp}\right]\right)}{C_{\rho}}, \quad J_{2}=\frac{\tilde{\mathbf{g}}\left(\mathcal{X}^{\perp},\left[\mathcal{X}, \mathcal{X}^{\perp}\right]\right)}{C_{\rho}} .
$$

Identities $\tilde{\mathbf{g}}\left(\mathcal{X},\left[\mathcal{X}, \mathcal{X}^{\perp}\right]\right)=-\mathcal{X}^{\perp}\left(C_{\rho}\right)$ and $\tilde{\mathbf{g}}\left(\mathcal{X}^{\perp},\left[\mathcal{X}, \mathcal{X}^{\perp}\right]\right)=X^{\perp} C_{\rho}-C_{\rho} C_{v}$ are routinely checked in adapted coordinates. 


\section{$7 \wedge$-vacuum Einstein equations for $\boldsymbol{G}_{2}$ metrics, and their solutions in the special cases $C_{\rho}=0$ and $\ell_{\mathcal{C}}=0$}

Vacuum Einstein equations for metrics with two commuting Killing fields have been derived by Geroch [14,15], Gaffet [13, eq. (3.15)], Whelan and Romano [38]. Here we look for $\Lambda$-vacuum equations. We obtain a tractable system by choosing $\tilde{g}_{i j}, f_{j}^{k}, h_{k l}$ as dependent variables, i.e., substituting

$$
f_{i l}=f_{i}^{k} h_{k l}, \quad g_{i j}=\tilde{g}_{i j}+f_{i}^{k} f_{j}^{l} h_{k l} .
$$

This choice ensures that the components of the inverse matrix $\mathbf{g}^{\alpha \beta}$ are relatively simple. Then, to simplify the Einstein equations further, we exploit the fact that the metric $\tilde{g}$, being two-dimensional and non-degenerate, is conformally flat. Hence, depending on the position of the Killing leaves in the spacetime, $\tilde{g}$ is either conformally Euclidean or conformally Minkowskian. In addition, denoting by $H$ the symmetric $2 \times 2$ matrix with elements $h_{k l}$, it is useful to introduce the row vectors

$$
\begin{aligned}
F_{i} & =\left(f_{i}^{1} f_{i}^{2}\right), \quad i=1,2, \\
P & =\left(F_{1,2}-F_{2,1}\right) H,
\end{aligned}
$$

i.e., $P$ is a row vector obtained by multiplication of the row vector $F_{1,2}-F_{2,1}$ by $H$ from the right. By comparison with formula (20), $P=0$ iff $\mathcal{C}=0$ iff the metric is orthogonally transitive.

Below we derive the $\Lambda$-vacuum Einstein equations for $\mathrm{G}_{2}$-metrics. We find their explicit solutions in the special cases $C_{\rho}=0$ and $\ell_{\mathcal{C}}=0$. In particular, we show that when $C_{\rho}=0$, then the corresponding $\Lambda$-vacuum Einstein metrics belong to the well-understood class of $p p$ waves, characterized by the presence of a constant null vector, see [35, §25.5] and references therein. In this special case all first-order invariants vanish. In the case when $\ell_{\mathcal{C}}=0$, on the other hand, we show that the explicit vacuum solution originally presented by Kundu [21] can be extended to the $\Lambda$-vacuum case. In particular, we find two new solutions (43-44) and $(45-46)$.

\subsection{The case when $\tilde{\mathbf{g}}$ is Lorentzian and explicit solutions with $C_{\rho}=0$}

Proposition 12 Let the metric (10) be such that $C_{\rho} \neq 0$, with $\operatorname{det} \tilde{\mathbf{g}}<0$ and $\operatorname{det} H>0$. Then, by writing the orbit metric in the conformally flat form

$$
\tilde{\mathbf{g}}=\left(\begin{array}{ll}
0 & q \\
q & 0
\end{array}\right), \quad q=q\left(t^{1}, t^{2}\right) \neq 0,
$$

the $\Lambda$-vacuum Einstein equations $\mathbf{R}_{\mu \nu}-\Lambda \mathbf{g}_{\mu \nu}=0$ are equivalent to the compatible system of matrix and scalar equations

$$
\begin{aligned}
\left(r H_{, 1} H^{-1}\right)_{, 2}+\left(r H_{, 2} H^{-1}\right)_{, 1} & =2 \Lambda q r E+\frac{q}{r} A^{\top} A H^{-1}, \\
(\ln q)_{, 1} & =\left(\ln (\ln r)_{, 1}\right)_{, 1}+\frac{\operatorname{tr}\left(H_{, 1} H^{-1} H_{, 1} H^{-1}\right)}{4(\ln r)_{, 1}}, \\
(\ln q)_{, 2} & =\left(\ln (\ln r)_{, 2}\right)_{, 2}+\frac{\operatorname{tr}\left(H_{, 2} H^{-1} H_{, 2} H^{-1}\right)}{4(\ln r)_{, 2}}, \\
F_{1,2}-F_{2,1} & =\frac{q}{r} A H^{-1},
\end{aligned}
$$


where $r=\sqrt{\operatorname{det} H}, E$ is the $2 \times 2$ unit matrix and

$$
A=\left(a_{1} a_{2}\right), \quad a_{i}=\text { const }
$$

denotes an arbitrary constant row vector, which is zero if and only if $\Xi^{\perp}$ is completely integrable. Moreover,

$$
r_{, 12}=-\Lambda q r-\frac{q}{4 r} A H^{-1} A^{\top}
$$

as a consequence of first equation of (27).

Proof By assumption $C_{\rho} \neq 0$, where

$$
C_{\rho}=8 \frac{r_{, 1} r_{, 2}}{r^{2} q}=2 \frac{(\operatorname{det} H)_{, 1}(\operatorname{det} H)_{, 2}}{(\operatorname{det} H)^{2} q} .
$$

Therefore, $(\operatorname{det} H)_{, 1} \neq 0,(\operatorname{det} H)_{, 2} \neq 0$. Denote $\mathbf{R}$ the Ricci tensor of the metric $\mathbf{g}$. Solving the Einstein equations $\mathbf{R}_{\mu \nu}-\Lambda \mathbf{g}_{\mu \nu}$ with respect to $H_{, 12}, P_{, 1}, P_{, 2}, q_{, 1}, q_{, 2}, q_{, 12}$, we obtain one $2 \times 2$ matrix equation

$$
\begin{aligned}
H_{, 12} & -\frac{1}{2} H_{, 1} H^{-1} H_{, 2}-\frac{1}{2} H_{, 2} H^{-1} H_{, 1} \\
& +\frac{1}{4} \frac{(\operatorname{det} H)_{, 1}}{\operatorname{det} H} H_{, 2}+\frac{1}{4} \frac{(\operatorname{det} H)_{, 2}}{\operatorname{det} H} H_{, 1}+\Lambda q H+\frac{1}{2 q} P^{\top} P=0,
\end{aligned}
$$

two vector equations

$$
\begin{aligned}
P_{, 1} & =\left(\frac{(\operatorname{det} H)_{, 11}}{(\operatorname{det} H)_{, 1}}-\frac{\operatorname{det}\left(H_{, 1}\right)}{(\operatorname{det} H)_{, 1}}-\frac{(\operatorname{det} H)_{, 1}}{\operatorname{det} H}\right) P, \\
P_{, 2} & =\left(\frac{(\operatorname{det} H)_{, 22}}{(\operatorname{det} H)_{, 2}}-\frac{\operatorname{det}\left(H_{, 2}\right)}{(\operatorname{det} H)_{, 2}}-\frac{(\operatorname{det} H)_{, 2}}{\operatorname{det} H}\right) P,
\end{aligned}
$$

and three scalar equations

$$
\begin{aligned}
q_{, 1} & =\left(\frac{(\operatorname{det} H)_{, 11}}{(\operatorname{det} H)_{, 1}}-\frac{\operatorname{det}\left(H_{, 1}\right)}{(\operatorname{det} H)_{, 1}}-\frac{1}{2} \frac{(\operatorname{det} H)_{, 1}}{\operatorname{det} H}\right) q, \\
q_{, 2} & =\left(\frac{(\operatorname{det} H)_{, 22}}{(\operatorname{det} H)_{, 2}}-\frac{\operatorname{det}\left(H_{, 2}\right)}{(\operatorname{det} H)_{, 2}}-\frac{1}{2} \frac{(\operatorname{det} H)_{, 2}}{\operatorname{det} H}\right) q, \\
(\ln q)_{, 12}= & \frac{(\operatorname{tr} H)_{, 1}(\operatorname{tr} H)_{, 2}-\operatorname{tr}\left(H_{, 1} H_{, 2}\right)}{4 \operatorname{det} H}+\frac{3}{4 q} P H^{-1} P^{\top} .
\end{aligned}
$$

By comparison of the cross derivatives $q, 12$ and $q, 21$, one sees that the third equation (30) is a differential consequence of the first two.

Conversely, if the five equations (28), (29) and (30) hold, then $\mathbf{R}_{\mu \nu}=\Lambda \mathbf{g}_{\mu \nu}$. Compatibility of the equations is routinely checked.

As an easy consequence of equations (29) and (30), we obtain

$$
P_{, 1}=\left(\frac{q, 1}{q}-\frac{1}{2} \frac{(\operatorname{det} H)_{, 1}}{\operatorname{det} H}\right) P, \quad P_{, 2}=\left(\frac{q, 2}{q}-\frac{1}{2} \frac{(\operatorname{det} H)_{, 2}}{\operatorname{det} H}\right) P .
$$

It follows that $r P / q$ is a constant vector (recall that $r=\sqrt{\operatorname{det} H}$ ). Therefore, we can write

$$
P=\frac{q}{r} A,
$$

where $A$ is an arbitrary constant row vector. Now the Einstein equations reduce to system (27). 
Remark 4 When $A=0$ and $\Lambda=0$, equations (27) reduce to the well-known BelinskyZakharov [4] formulation of the vacuum Einstein equations.

Proposition 13 All $\Lambda$-vacuum Einstein metrics of the form (10), with $C_{\rho}=0$, $\operatorname{det} \tilde{\mathbf{g}}<0$ and $\operatorname{det} H>0$, satisfy $\Lambda=0$ and in adapted coordinates can be written in the form

$$
\mathbf{g}=d t^{1} d t^{2}+R^{2}\left(d z^{1}+W d z^{2}\right)^{2}+S^{2}\left(d z^{2}\right)^{2},
$$

with $R, W$ and $S$ differentiable functions of $t^{1}$ such that $R S \neq 0$ and

$$
\left(W^{\prime}\right)^{2}=\frac{2 S^{2}}{R^{2}}\left(\frac{R^{\prime \prime}}{R}+\frac{S^{\prime \prime}}{S}\right) .
$$

In particular these Ricci-flat metrics are such that $\mathcal{C}=0$ (then $\left.\ell_{\mathcal{C}}=0\right)$, hence are orthogonally transitive and, in addition, are pp-waves since $\partial_{t^{2}}$ is a null Killing vector field such that $\nabla \partial_{t^{2}}=0$.

Proof By assumption

$$
0=C_{\rho}=2 \frac{(\operatorname{det} H)_{, 1}(\operatorname{det} H)_{, 2}}{(\operatorname{det} H)^{2} q} .
$$

Therefore, det $H$ is a function of either $t^{1}$ or $t^{2}$; we assume the former, i.e., $t^{1}$. On the other hand, since $h_{11} \neq 0$ can be always achieved by a linear change of coordinates $\left\{\bar{z}^{i}=\alpha_{j}^{i} z^{j}\right\}$, without loss of generality one can write $\mathbf{h}$ (the restriction of the metric to the Killing leaves $\Xi)$ as

$$
\mathbf{h}=h_{11}\left[\left(d z^{1}+\frac{h_{12}}{h_{11}} d z^{2}\right)^{2}+\frac{\left(h_{11} h_{22}-h_{12}^{2}\right)}{h_{11}^{2}}\left(d z^{2}\right)^{2}\right],
$$

i.e., in the Weyl-Lewis-Papapetrou form [22,31]

$$
\mathbf{h}=\frac{r}{s}\left[\left(d z^{1}+w d z^{2}\right)^{2}+s^{2}\left(d z^{2}\right)^{2}\right]
$$

with

$$
w=\frac{h_{12}}{h_{11}}, \quad r=\sqrt{\operatorname{det} H}, \quad s=\frac{r}{h_{11}} .
$$

In terms of Weyl-Lewis-Papapetrou parameters $r, s, w$, the analysis of Einstein equations $\mathbf{L}_{\mu \nu}=\mathbf{R}_{\mu \nu}-\Lambda \mathbf{g}_{\mu \nu}=0$ simplifies noteworthy. Indeed, by computing the contravariant components $L^{\mu \nu}$, one finds that $\mathbf{L}^{11}=0$ if and only if $s_{, 2}^{2}+w_{, 2}^{2}=0$. Since $w, s$ are real, it follows that they are functions of $t^{1}$. Hence, all components $h_{i j}$ are functions of $t^{1}$, which substantially simplifies computation of the remaining components of $\mathbf{L}$. In particular, we obtain

$$
0=\mathbf{L}^{13}+f_{2}^{1} \mathbf{L}^{12}=-\frac{1}{2} \mathcal{C}_{t^{2}}^{3}, \quad 0=\mathbf{L}^{14}+f_{2}^{2} \mathbf{L}^{12}=-\frac{1}{2} \mathcal{C}_{t^{2}}^{4}
$$


Consequently, components of the curvature vector depend on $t^{1}$ only as well. Continuing further, we obtain

$$
\begin{aligned}
0 & =\mathbf{L}_{33}+3 h_{11} \mathbf{L}^{12}=\frac{1}{2}\left(\mathcal{C}^{2}\right)^{2} \operatorname{det} H+\left(\frac{q, 12}{q^{3}}-\frac{q, 1 q, 2}{q^{2}}\right) h_{11}+4 \Lambda h_{11}, \\
0 & =\mathbf{L}_{34}+3 h_{12} \mathbf{L}^{12}=\frac{1}{2} \mathcal{C}^{3} \mathcal{C}^{2} \operatorname{det} H+\left(\frac{q, 12}{q^{3}}-\frac{q, 1 q, 2}{q^{2}}\right) h_{12}+4 \Lambda h_{12}, \\
0 & =\mathbf{L}_{44}+3 h_{22} \mathbf{L}^{12}=\frac{1}{2}\left(\mathcal{C}^{3}\right)^{2} \operatorname{det} H+\left(\frac{q, 12}{q^{3}}-\frac{q, 1 q, 2}{q^{2}}\right) h_{22}+4 \Lambda h_{22} \\
0 & =(4 q \operatorname{det} H) \mathbf{L}^{12}+h_{22} \mathbf{L}_{33}-2 h_{12} \mathbf{L}_{34}+h_{11} \mathbf{L}_{44} \\
& =2\left(\frac{q, 12}{q^{3}}-\frac{q, 1 q_{, 2}}{q^{2}}+3 \Lambda\right) \operatorname{det} H
\end{aligned}
$$

By the fourth equation,

$$
\frac{q, 12}{q^{3}}-\frac{q, 1 q, 2}{q^{2}}=-3 \Lambda
$$

then, by substituting into the remaining three equations and using $\left(\mathcal{C}^{3}\right)^{2}\left(\mathcal{C}^{2}\right)^{2}=\left(\mathcal{C}^{3} \mathcal{C}^{2}\right)^{2}$, we obtain $\Lambda=0$ and

$$
q, 12=\frac{q, 1 q, 2}{q} .
$$

Hence, $\mathcal{C}=0$ and the metric $\mathbf{g}$ is orthogonally transitive.

On the other hand, Eq. (33) implies that $q\left(t^{1}, t^{2}\right)$ is a product, $q=q_{1}\left(t^{1}\right) q_{2}\left(t^{2}\right)$. Therefore, by passing to new coordinates $\bar{t}^{i}=\int q_{i} d t^{i}$, the orbit metric reduces to $d \bar{t}^{2} d \bar{t}^{2}$ and the Einstein equations reduce to a single ordinary differential equation. Hence, by suitably rearranging the unknown functions, one can write the metric $\mathbf{g}$ and the corresponding Einstein equations in the form (31) and (32), respectively. The case when det $H$ depends on $t^{2}$ is completely analogous.

Obviously, the three Killing fields commute and, therefore, the metric has no unique twodimensional commuting Killing algebra. Hence, it actually falls outside the class of metrics considered in this paper.

\subsection{The case when $\tilde{\mathrm{g}}$ is Riemannian and explicit solutions with $C_{\rho}=0$}

In the case of conformally Euclidean orbit metric, we have $\tilde{g}=q\left(\mathrm{~d} t^{1}\right)^{2}+q\left(\mathrm{~d} t^{2}\right)^{2}$ and, therefore,

$$
\mathbf{g}=q\left(\left(\mathrm{~d} t^{1}\right)^{2}+\left(\mathrm{d} t^{2}\right)^{2}\right)+h_{k l}\left(\mathrm{~d} z^{k}+f_{1}^{k} \mathrm{~d} t^{1}+f_{2}^{k} \mathrm{~d} t^{2}\right)\left(\mathrm{d} z^{l}+f_{1}^{l} \mathrm{~d} t^{1}+f_{2}^{l} \mathrm{~d} t^{2}\right),
$$

where $q, f_{i}^{k}, h_{k l}$ are the unknown functions of $x, y$. Clearly, det $H<0$.

Proposition 14 Let the metric (10) be such that $C_{\rho} \neq 0$, with $\operatorname{det} \tilde{\mathbf{g}}>0$ and $\operatorname{det} H<0$. Then, by writing the orbit metric in the conformally flat form

$$
\tilde{\mathbf{g}}=\left(\begin{array}{ll}
q & 0 \\
0 & q
\end{array}\right), \quad q=q\left(t^{1}, t^{2}\right) \neq 0 .
$$


the $\Lambda$-vacuum Einstein equations $\mathbf{R}_{\mu \nu}-\Lambda \mathbf{g}_{\mu \nu}=0$ are equivalent to the compatible system of matrix and scalar equations

$$
\begin{aligned}
& \left(r H_{, 1} H^{-1}\right)_{, 1}+\left(r H_{, 2} H^{-1}\right)_{, 2}=2 \Lambda q r E+\frac{q}{r} A^{\top} A H^{-1}, \\
& \left(\ln \frac{q}{r_{, 1}^{2}+r_{, 2}^{2}}\right)_{, 1}=-\frac{r_{, 11}+r_{, 22}}{r_{, 1}^{2}+r_{, 2}^{2}} r_{, 1}+\frac{\operatorname{det}\left(H_{, 1}\right)-\operatorname{det}\left(H_{, 2}\right)}{r_{, 1}^{2}+r_{, 2}^{2}} \frac{r_{, 1}}{2 r} \\
& +\frac{(\operatorname{tr} H)_{, 1}(\operatorname{tr} H)_{, 2}-\operatorname{tr}\left(H_{, 1} H_{, 2}\right)}{r_{, 1}^{2}+r_{, 2}^{2}} \frac{r, 2}{2 r}, \\
& \left(\ln \frac{q}{r_{, 1}^{2}+r_{, 2}^{2}}\right)_{, 2}=-\frac{r_{, 11}+r_{, 22}}{r_{, 1}^{2}+r_{, 2}^{2}} r_{, 2}+\frac{\operatorname{det}\left(H_{, 2}\right)-\operatorname{det}\left(H_{, 1}\right)}{r_{, 1}^{2}+r_{, 2}^{2}} \frac{r_{, 2}}{2 r} \\
& +\frac{(\operatorname{tr} H)_{, 1}(\operatorname{tr} H)_{, 2}-\operatorname{tr}\left(H_{, 1} H_{, 2}\right)}{r_{, 1}^{2}+r_{, 2}^{2}} \frac{r_{, 1}}{2 r}, \\
& F_{1,2}-F_{2,1}=\frac{q}{r} A H^{-1}
\end{aligned}
$$

where $r=\sqrt{-\operatorname{det} H}, E$ is the unit $2 \times 2$ matrix and

$$
A=\left(a_{1} a_{2}\right), \quad a_{i}=\text { const }
$$

is an arbitrary constant row vector, which is zero if and only if $\Xi^{\perp}$ is completely integrable. Moreover,

$$
r_{, 11}+r_{, 22}=-2 \Lambda q r+\frac{q}{2 r} A H^{-1} A^{\top}
$$

as a consequence of the first equation of (35).

Proof By assumption

$$
0 \neq C_{\rho}=4 \frac{r_{, 1}^{2}+r_{, 2}^{2}}{q r^{2}}
$$

Consequently, also $r_{, 1}^{2}+r, 2^{2} \neq 0$. Denote $\mathbf{R}$ the Ricci tensor of the metric (34). By tedious routine computations, solving the Einstein equations $\mathbf{R}_{\mu \nu}-\Lambda \mathbf{g}_{\mu \nu}$ with respect to $H_{, 22}, P_{, 1}, P_{, 2}, q_{, 1}, q_{, 2}, q_{, 22}$, we obtain one $2 \times 2$ matrix equation

$$
\begin{aligned}
& H_{, 11}+H_{, 22}-H_{, 1} H^{-1} H_{, 1}-H_{, 2} H^{-1} H_{, 2} \\
& \quad+\frac{1}{2} \frac{(\operatorname{det} H)_{, 1}}{\operatorname{det} H} H_{, 1}+\frac{1}{2} \frac{(\operatorname{det} H)_{, 2}}{\operatorname{det} H} H_{, 2}+2 \Lambda q H+\frac{1}{q} P^{\top} P=0,
\end{aligned}
$$

two vector equations

$$
P_{, 1}=\left(\frac{q, 1}{q}-\frac{1}{2} \frac{(\operatorname{det} H)_{, 1}}{\operatorname{det} H}\right) P, \quad P_{, 2}=\left(\frac{q, 2}{q}-\frac{1}{2} \frac{(\operatorname{det} H)_{, 2}}{\operatorname{det} H}\right) P,
$$


and three scalar equations

$$
\begin{aligned}
& (\operatorname{det} H)_{, 2} \frac{q, 1}{q}+(\operatorname{det} H)_{, 1} \frac{q, 2}{q}-2(\operatorname{det} H)_{, 12} \\
& +\frac{(\operatorname{det} H)_{, 1}(\operatorname{det} H)_{, 2}}{\operatorname{det} H}+(\operatorname{tr} H)_{, 1}(\operatorname{tr} H)_{, 2}-\operatorname{tr}\left(H_{, 1} H_{, 2}\right)=0, \\
& (\operatorname{det} H)_{, 1} \frac{q, 1}{q}-(\operatorname{det} H)_{, 2} \frac{q, 2}{q}+(\operatorname{det} H)_{, 11}-(\operatorname{det} H)_{, 22} \\
& \quad-\frac{(\operatorname{det} H)_{, 1}^{2}+(\operatorname{det} H)_{, 2}^{2}}{2 \operatorname{det} H}-\operatorname{det}\left(H_{, 1}\right)+\operatorname{det}\left(H_{, 2}\right)=0, \\
& (\ln q)_{, 11}+(\ln q)_{, 22}=\frac{\operatorname{det}\left(H_{, 1}\right)+\operatorname{det}\left(H_{, 2}\right)}{2 \operatorname{det} H}-\frac{3}{4 q} P H^{-1} P^{\top} .
\end{aligned}
$$

Again, the third equation (38) is a differential consequence of the first two.

Conversely, if the five equations (36), (37) and (38) hold, then $\mathbf{R}_{\mu \nu}=\Lambda \mathbf{g}_{\mu \nu}$. Compatibility of the equations is routinely checked. Again, $r P / q$ is a constant vector (recall that $r=$ $\sqrt{-\operatorname{det} H}$ ) and we can write

$$
P=\frac{q}{r} A,
$$

where $A$ is a constant row vector.

The two scalar equations (38) simplify to

$$
\begin{aligned}
r_{, 2} \frac{q, 1}{q}+r_{, 1} \frac{q, 2}{q}-2 r_{, 12}-\frac{(\operatorname{tr} H)_{, 1}(\operatorname{tr} H)_{, 2}}{2 r}+\frac{\operatorname{tr}\left(H_{, 1} H_{, 2}\right)}{2 r}=0, \\
-r_{, 1} \frac{q, 1}{q}+r_{, 2} \frac{q, 2}{q}+r_{, 11}-r_{, 22}+\frac{\operatorname{det}\left(H_{, 2}\right)-\operatorname{det}\left(H_{, 2}\right)}{2 r}=0 .
\end{aligned}
$$

Then, the Einstein equations reduce to system (35).

Proposition 15 All $\Lambda$-vacuum Einstein metrics of the form (10), with $C_{\rho}=0$, $\operatorname{det} \tilde{\mathbf{g}}>0$ and $\operatorname{det} H<0$, satisfy $\Lambda=0$ and in adapted coordinates can be written either in the form

$$
\mathbf{g}=\left(d t^{1}\right)^{2}+\left(d t^{2}\right)^{2}+\psi\left(d z^{1}\right)^{2}+2\left(c t^{1} d t^{2}+d z^{2}\right) d z^{1},
$$

with $c \in \mathbb{R}$ and $\psi=\psi\left(t^{1}, t^{2}\right)$ a differentiable function such that $\psi, 11+\psi, 22=c^{2}$, or in the form

$$
\mathbf{g}=e^{t^{1}}\left(d t^{1}\right)^{2}+e^{t^{1}}\left(d t^{2}\right)^{2}+\psi\left(d z^{1}\right)^{2}+2\left(c e^{t^{1}} d t^{2}+d z^{2}\right) d z^{1}
$$

with $c \in \mathbb{R}$ and $\psi=\psi\left(t^{1}, t^{2}\right)$ a differentiable function such that $\psi_{, 11}+\psi_{, 22}=e^{t^{1}} c^{2}$.

In particular, these Ricci-flat metrics are such that $\ell_{\mathcal{C}}=0$ and, in addition, are pp-waves since $\partial_{z^{2}}$ is a null Killing vector field such that $\nabla \partial_{z^{2}}=0$; moreover, these metrics are orthogonally transitive if, and only if, $c=0$.

Proof By assumption

$$
0=C_{\rho}=\frac{\left(\operatorname{det} H_{, 1}\right)^{2}+\left(\operatorname{det} H_{, 2}\right)^{2}}{q(\operatorname{det} H)^{2}} .
$$

Consequently, det $H$ is a constant. Like in the proof of Proposition 13, the analysis of Einstein equations $\mathbf{L}_{\mu v}=\mathbf{R}_{\mu \nu}-\Lambda \mathbf{g}_{\mu \nu}=0$ simplifies noteworthy if considering $\mathbf{h}$ in the WeylLewis-Papapetrou form. In terms of the Weyl-Lewis-Papapetrou parameters $r, s, w$, one 
has det $H=-r^{2}$ and, without loss in generality, one can assume $r=1$, because this can always be achieved by a coordinate transformation $z^{i} \rightarrow z^{i} / \sqrt{|r|}, i=1,2$, and rearranging the sign of $s$ whenever $r<0$. Moreover, by equating contravariant components $\mathbf{L}^{12}$ and $\mathbf{L}^{11}-\mathbf{L}^{22}$ to zero modulo det $H=$ const, we obtain

$$
\begin{aligned}
& 0=h_{11,2} h_{22,1}-2 h_{12,1} h_{12,2}+h_{11,1} h_{22,2}=s_{, 1} s_{, 2}-w_{, 1} w_{, 2}, \\
& 0=h_{11,1} h_{22,1}-h_{12,1^{2}}{ }^{2} h_{12,2}{ }^{2}-h_{11,2} h_{22,2}=s_{, 1}{ }^{2}-s_{, 2}{ }^{2}-w_{, 1}{ }^{2}+w_{, 2}{ }^{2},
\end{aligned}
$$

The latter algebraic system has two real solutions

$$
s_{, 1}= \pm w_{, 1}, \quad s_{, 2}= \pm w_{, 2}
$$

and also a complex solution $s_{, 2}=\mathrm{i} w_{, 1}, s_{, 1}=-\mathrm{i} w_{, 2}$, which gives $s=$ const, $w=$ const as the unique real subcase. Altogether, we obtain

$$
s= \pm w+c_{1}, \quad r=1,
$$

Hence,

$$
H=\left(\begin{array}{cc}
\frac{1}{ \pm w+c_{1}} & \frac{w}{ \pm w+c_{1}} \\
\frac{w}{ \pm w+c_{1}} & \frac{-c_{1}^{2} \mp 2 c_{1} w}{ \pm w+c_{1}}
\end{array}\right) \sim\left(\begin{array}{cc}
\frac{1}{ \pm w+c_{1}} & 1 \\
1 & 0
\end{array}\right)
$$

where the matrix congruence $H \sim Q^{\top} H Q$ is with respect to the transition matrix

$$
Q=\left(\begin{array}{cc} 
\pm 1 & \pm c_{1} \\
0 & 1
\end{array}\right)
$$

Otherwise said, we can take

$$
H=\left(\begin{array}{cc}
\psi & 1 \\
1 & 0
\end{array}\right)
$$

with $\psi=\psi\left(t^{1}, t^{2}\right)$ a differentiable function. This simplifies $\mathbf{L}$ further. From $\mathbf{L}^{44}=0$ we get $\mathcal{C}^{3}=0$, i.e., $f_{1,2}^{1}-f_{2,1}^{1}=0$, and by $\mathbf{L}_{34}=0$ we get $\Lambda=0$. Then, $\mathbf{L}^{11}=0$ is equivalent to $q, 11+q, 22=\left(q_{, 1}^{2}+q_{, 2}^{2}\right) / q$, which transforms to the Laplace equation $\phi_{, 11}+\phi_{, 22}=0$ under $q=e^{\phi}$, and by $L^{14}=L^{24}=0$ one gets that $\mathcal{C}^{4}=$ const, i.e., $f_{2,1}^{2}-f_{1,2}^{2}=c e^{\phi}$, with $c \in \mathbb{R}$. It follows that the remaining equations $L^{\mu \nu}=0$ are satisfied if, and only if,

$$
\psi, 11+\psi, 22=c^{2} e^{\phi} .
$$

Thus, when $\phi=c_{0}, c_{0} \in \mathbb{R}$, one has

$$
f_{1}^{1}=\phi_{21,1}+\phi_{1}, \quad f_{1}^{2}=\phi_{12,1}, \quad f_{2}^{1}=\phi_{21,2}, \quad f_{2}^{2}=\phi_{12,2}+c e^{c_{0}} t^{1}+\phi_{2},
$$

with $\phi_{12}=\phi_{12}\left(t^{1}, t^{2}\right), \phi_{21}=\phi_{21}\left(t^{1}, t^{2}\right), \phi_{1}=\phi_{1}\left(t^{1}\right), \phi_{2}=\phi_{2}\left(t^{2}\right)$ arbitrary differentiable functions and, by choosing new adapted coordinates

$$
\bar{t}^{1}=e^{c_{0} / 2} t^{1}, \quad \bar{t}^{2}=e^{c_{0} / 2} t^{2}, \quad \bar{z}^{1}=\phi_{21}+\phi_{1}+z^{1}, \quad \bar{z}^{2}=\phi_{12}+\phi_{2}+z^{2},
$$

we get

$$
\mathbf{g}=\left(d \bar{t}^{1}\right)^{2}+\left(d \bar{t}^{2}\right)^{2}+\psi\left(d \bar{z}^{1}\right)^{2}+2\left(c \bar{t}^{-1} d \bar{t}^{2}+d \bar{z}^{2}\right) d \bar{z}^{1},
$$

where $\partial_{\bar{t}^{1}}^{2} \psi+\partial_{\bar{t}^{2}}^{2} \psi=c^{2}$. 
On the other hand, if $\phi$ is non-constant, then, being a harmonic function, $\phi$ can be chosen for $t^{1}$ and the conjugate harmonic function for $t^{2}$. Then,

$$
f_{1}^{1}=\phi_{21,1}+\phi_{1}, \quad f_{1}^{2}=\phi_{12,1}, \quad f_{2}^{1}=\phi_{21,2}, \quad f_{2}^{2}=\phi_{12,2}+c e^{t^{1}}+\phi_{2},
$$

with $\phi_{12}=\phi_{12}\left(t^{1}, t^{2}\right), \phi_{21}=\phi_{21}\left(t^{1}, t^{2}\right), \phi_{1}=\phi_{1}\left(t^{1}\right), \phi_{2}=\phi_{2}\left(t^{2}\right)$ arbitrary differentiable functions. By choosing new adapted coordinates

$$
\bar{t}^{1}=t^{1}, \quad \bar{t}^{2}=t^{2}, \quad \bar{z}^{1}=\phi_{21}+\phi_{1}+z^{1}, \quad \bar{z}^{2}=\phi_{12}+\phi_{2}+z^{2},
$$

we get

$$
\mathbf{g}=e^{\bar{t}^{1}}\left(d \bar{t}^{1}\right)^{2}+e^{\bar{t}^{1}}\left(d \bar{t}^{2}\right)^{2}+\psi\left(d \bar{z}^{1}\right)^{2}+2\left(c e^{\bar{t}^{1}} d \bar{t}^{2}+d \bar{z}^{2}\right) d \bar{z}^{1},
$$

where $\partial_{\bar{t}^{1}}^{2} \psi+\partial_{\bar{t}^{2}}^{2} \psi=e^{\bar{t}^{1}} c^{2}$. These Ricci-flat metrics are $p p$-waves since $\partial / \partial \bar{z}^{2}$ is a covariantly constant and null Killing vector.

\subsection{Exact solutions in the case when $\ell_{\mathcal{C}}=0$}

In the paper [21] Kundu looked for solutions of the vacuum Einstein equations satisfying the condition $h^{k l} c_{k} c_{l}=0$, where the scalars

$$
c_{i}=\epsilon^{\alpha \beta \rho \sigma} \xi_{(1) \alpha} \xi_{(2) \beta} \xi_{(i) \rho ; \sigma}
$$

measure the orthogonal intransitivity (cf. [14]), and $\xi_{(i)}=\partial_{z^{i}}, i=1,2$, are the Killing vectors. Kundu presented all solutions satisfying this condition, but without proof. We reconstruct the proof below and extend his result to $\Lambda$-vacuum metrics.

To start with, we notice that the Kundu condition $h^{k l} c_{k} c_{l}=0$ is equivalent to $\mathbf{c}_{12}^{\alpha} \mathbf{c}_{12}^{\beta} \mathbf{g}_{\alpha \beta}=$ $\mathbf{c}_{12}^{k^{*}} \mathbf{c}_{12}^{*} h_{k l}=0$, i.e., in invariant terms,

$$
\ell_{\mathcal{C}}=0
$$

Lemma 4 When $C_{\rho} \neq 0$, the semi-invariant vector field $\mathcal{C}$ and the invariant $\ell_{\mathcal{C}}$ can be written as

$$
\mathcal{C}=\operatorname{sgn}(q) \frac{1}{\sqrt{\mp \operatorname{det} H}} A H^{-1}, \quad \ell_{\mathcal{C}}=\frac{1}{\mp \operatorname{det} H} A H^{-1} A^{\top},
$$

where $A$ is the constant vector introduced in Propositions 12 and 14. Moreover, under transformations $\partial / \partial z^{j}=\alpha_{j}^{i} \partial / \partial \bar{z}^{i}$, with $\left(\alpha_{j}^{i}\right) \in \mathrm{GL}(2, \mathbb{R})$, we have

$$
H \rightarrow \alpha^{\top} H \alpha, \quad A \rightarrow A \alpha, \quad q \rightarrow q, \quad P \rightarrow P
$$

and, whenever $A$ is nonzero, it can be always normalized to any prescribed nonzero vector by means of transformation (41).

Proof This is easily checked using Propositions 12 and 14.

The next theorem describes all $\Lambda$-vacuum Einstein metrics with $\ell_{\mathcal{C}}=0$.

Theorem 1 Every Lorentzian $\Lambda$-vacuum metric of the form (10) that satisfies the condition $\ell_{\mathcal{C}}=0$ has one of the following forms:

1. pp-waves with $C_{\rho}=0$, described by Propositions 13 and 15; 
2. Petrov type II vacuum metrics of Kundu [21]

$$
\frac{1}{\sqrt{x}}\left(d x^{2}+d y^{2}+\left(x^{3 / 2} \psi+1\right) d u^{2}+2 d y d u-2 x^{3 / 2} d u d v\right),
$$

where $\psi$ solves the cylindrical Laplace equation $\psi_{x x}-2 \psi_{x} / x+\psi_{y y}=0$;

3. Petrov type II $\Lambda$-vacuum metrics

$$
\frac{3}{\Lambda} \frac{c^{2} r}{c^{2} r^{3}+1} d r^{2}+\frac{c^{2} r^{3}+1}{r} d y^{2}+\frac{2}{r} d y d u+\frac{r^{3} \psi+1}{r} d u^{2}+\frac{2}{r^{2}} d u d v
$$

where $c, \Lambda$ are nonzero constants and $\psi(r, y)$ is a solution of the separable linear equation

$$
\frac{\left(c^{2} r^{3}+1\right)^{2}}{r^{2}} \psi_{r r}+\frac{\left(c^{2} r^{3}+1\right)\left(4 c^{2} r^{3}+1\right)}{r^{3}} \psi_{r}+3 \frac{c^{2}}{\Lambda} \psi_{y y}=0
$$

4. Petrov type III $\Lambda$-vacuum metrics

$$
\frac{3}{\Lambda x^{2}} d x^{2}+\frac{1}{x^{2}} d y^{2}+2 x d y d u+\frac{2}{x^{2}} d u d v+\frac{x^{6}+\psi}{2 x^{2}} d u^{2}
$$

where $\Lambda \neq 0$ and $\psi(x, y)$ satisfy the cylindrical Laplace equation

$$
\psi_{x x}-\frac{2}{x} \psi_{x}+\frac{3}{\Lambda} \psi_{y y}=0 .
$$

Proof The case of $C_{\rho}=0$ is completed in Sect. 7, since all metrics found in Propositions 13 and 15 satisfy $\ell_{\mathcal{C}}=0$. Assume henceforth that $C_{\rho} \neq 0$, so that we can use Propositions 12 and 14

We consider the $\Lambda$-vacuum Einstein equations augmented with condition $\ell_{\mathcal{C}}=0$. According to Lemma 4 , the scalar invariant $\ell_{\mathcal{C}}$ equals

$$
\ell_{\mathcal{C}}=\frac{1}{\mp \operatorname{det} H} A H^{-1} A^{\top} .
$$

Therefore, condition $\ell_{\mathcal{C}}=0$ implies that the vector $\mathcal{C}$ is null with respect to the matrix $H^{-1}$; then necessarily det $H<0$, so that Proposition 12 is applicable. Rewriting $\Lambda$-vacuum Einstein equations in terms of Weyl-Lewis-Papapetrou parameters and renaming $t^{1}, t^{2}$ to $x, y$ for brevity, we obtain

$$
\begin{aligned}
r_{x x} & =\frac{1}{4} \frac{r_{x}^{2}-r_{y}^{2}}{r}+\frac{1}{4} r \frac{w_{x}^{2}-w_{y}^{2}-s_{x}^{2}+s_{y}^{2}}{s^{2}}+\frac{1}{2} \frac{r_{x} q_{x}-r_{y} q_{y}}{q}+\frac{1}{4} r q \ell_{\mathcal{C}}-\Lambda r q, \\
r_{x y}= & \frac{1}{2} \frac{r_{x} r_{y}}{r}+\frac{1}{2} r \frac{w_{x} w_{y}-s_{x} s_{y}}{s^{2}}+\frac{1}{2} \frac{r_{x} q_{y}+r_{y} q_{x}}{q}, \\
r_{y y}= & \frac{1}{4} \frac{r_{y}^{2}-r_{x}^{2}}{r}+\frac{1}{4} r \frac{w_{y}^{2}-w_{x}^{2}-s_{y}^{2}+s_{x}^{2}}{s^{2}}+\frac{1}{2} \frac{r_{y} q_{y}-r_{x} q_{x}}{q}+\frac{1}{4} r q \ell_{\mathcal{C}}-\Lambda r q, \\
q_{x x}+q_{y y}= & \frac{q_{x}^{2}+q_{y}^{2}}{q}+\frac{1}{2} q \frac{r_{x}^{2}+r_{y}^{2}}{r^{2}}+\frac{1}{2} q \frac{w_{y}^{2}+w_{x}^{2}-s_{y}^{2}-s_{x}^{2}}{s^{2}}-\frac{3}{2} q^{2} \ell_{\mathcal{C}}, \\
s_{x x}+s_{y y}= & -\frac{r_{x} s_{x}+r_{y} s_{y}}{r}+\frac{w_{x}^{2}+w_{y}^{2}+s_{x}^{2}+s_{y}^{2}}{s} \\
& -\frac{1}{2} q \frac{a_{1}^{2}\left(s^{2}+w^{2}\right)-2 a_{1} a_{2} w+a_{2}^{2}}{r^{3}}, \\
w_{x x}+w_{y y}= & -\frac{r_{x} w_{x}+r_{y} w_{y}}{r}+2 \frac{w_{x} s_{x}+w_{y} s_{y}}{s}-\frac{s q}{r^{3}} a_{1}\left(a_{1} w-a_{2}\right),
\end{aligned}
$$


where

$$
\ell_{\mathcal{C}}=\frac{a_{1}^{2}\left(s^{2}-w^{2}\right)+2 a_{1} a_{2} w-a_{2}^{2}}{s r^{3}}=\frac{\left(a_{1} s-a_{1} w+a_{2}\right)\left(a_{1} s+a_{1} w-a_{2}\right)}{s r^{3}},
$$

according to Eq. (47). If system (48) is solved, then the components $f_{i}^{j}$ can be found from the underdetermined system

$$
f_{1, y}^{1}-f_{2, x}^{1}=\left(a_{1} s^{2}-a_{1} w^{2}+a_{2} w\right) \frac{q}{s r^{2}}, \quad f_{1, y}^{2}-f_{2, x}^{2}=\left(a_{1} w-a_{2}\right) \frac{q}{s r^{2}} .
$$

The condition $\ell_{\mathcal{C}}=0$ implies that $a_{1} \neq 0$, since otherwise $a_{1}=a_{2}=0$, contradicting the assumption that the metric is not orthogonally transitive. Then, one has $w= \pm s+a_{2} / a_{1}$. One can choose the upper sign without loss in generality since the equations are invariant with respect to the transformation $w \rightarrow-w, a_{2} \rightarrow-a_{2}, f_{i}^{2} \rightarrow-f_{i}^{2}$. Thus, we let

$$
w=s+\frac{a_{2}}{a_{1}} .
$$

Equations (48) turn into

$$
\begin{aligned}
r_{x x} & =\frac{1}{4} \frac{r_{x}^{2}-r_{y}^{2}}{r}+\frac{1}{2} \frac{r_{x} q_{x}-r_{y} q_{y}}{q}-\Lambda r q, \\
r_{x y} & =\frac{1}{2} \frac{r_{x} r_{y}}{r}+\frac{1}{2} \frac{r_{x} q_{y}+r_{y} q_{x}}{q}, \\
r_{y y} & =\frac{1}{4} \frac{r_{y}^{2}-r_{x}^{2}}{r}+\frac{1}{2} \frac{r_{y} q_{y}-r_{x} q_{x}}{q}-\Lambda r q, \\
q_{x x}+q_{y y} & =\frac{1}{2} q \frac{r_{x}^{2}+r_{y}^{2}}{r^{2}}+\frac{q_{x}^{2}+q_{y}^{2}}{q}, \\
s_{x x}+s_{y y} & =-\frac{r_{x} s_{x}+r_{y} s_{y}}{r}+2 \frac{s_{x}^{2}+s_{y}^{2}}{s}-\frac{a_{1}^{2} q s^{2}}{r^{3}},
\end{aligned}
$$

whence,

$$
r_{x x}+r_{y y}=-2 \Lambda r q .
$$

Moreover, the system (50) reduces to

$$
-f_{1, y}^{1}+f_{2, x}^{1}+a_{2} \frac{q}{r^{2}}=0, \quad-f_{1, y}^{2}+f_{2, x}^{2}-a_{1} \frac{q}{r^{2}}=0 .
$$

Now, system (51) is preserved under the coordinate transformations (isometries of the orbit metric)

$$
x \rightarrow \tilde{x}, \quad y \rightarrow \tilde{y}, \quad q \rightarrow \tilde{q}
$$

( $r, s, w$ being unchanged), where $\tilde{x}(x, y), \tilde{y}(x, y)$ are arbitrary functionally independent conjugate harmonic functions, i.e., $\tilde{x}_{x}=\tilde{y}_{y}, \tilde{x}_{y}=-\tilde{y}_{x}$, and

$$
\tilde{q}=\frac{q}{J}, \quad J=\frac{\partial(\tilde{x}, \tilde{y})}{\partial(x, y)}=\tilde{x}_{x}^{2}+\tilde{x}_{y}^{2} \neq 0 .
$$

In order to reproduce Kundu's result, assume that $\Lambda=0$. Then, $r$ is harmonic by Eq. (52). Moreover, $r$ is non-constant, since otherwise $C_{\rho}=8 r_{x} r_{y} / r^{2} q=0$, which we excluded at the beginning of the proof. Therefore, $r$ can be chosen for $\tilde{x}$. Transforming back to the coordinates $x, y$, we thus identify $r=x$. Next we put $a_{1}=3 / 2, s=1 /\left(S+x^{-3 / 2}\right)$, and 
$u=z^{1}, v=z^{2}$ to get solution (42), which is easily identified with the Kundu orthogonally intransitive solution [21, eq. (4), $\alpha=1]$.

To cover the remaining two cases, assume that $\Lambda \neq 0$. Then, we can express

$$
q=-\frac{r_{x x}+r_{y y}}{2 \Lambda r}
$$

and substitute back into system (51), obtaining two third-order equations on $r$. These are equivalent to

$$
\left(\frac{r_{x}^{2}+r_{y}^{2}}{r_{x x}+r_{y y}} r^{1 / 2}-\frac{2}{3} r^{3 / 2}\right)_{x}=0, \quad\left(\frac{r_{x}^{2}+r_{y}^{2}}{r_{x x}+r_{y y}} r^{1 / 2}-\frac{2}{3} r^{3 / 2}\right)_{y}=0,
$$

i.e.,

$$
r^{1 / 2}\left(r_{x}^{2}+r_{y}^{2}\right)=\left(\frac{2}{3} r^{3 / 2}+c\right)\left(r_{x x}+r_{y y}\right),
$$

where $c$ is an arbitrary constant. Equation (54) is equivalent to

$$
\rho_{x x}+\rho_{y y}=0
$$

under substitution $\rho=\rho(r)$, where $\rho(r)$ satisfies

$$
\left(\frac{2}{3} r^{3 / 2}+c\right) \frac{\partial^{2} \rho}{\partial r^{2}}+r^{1 / 2} \frac{\partial \rho}{\partial r}=0 .
$$

The last equation is easily integrated,

$$
\rho=\int \frac{d r}{r^{3 / 2}+c},
$$

which yields $r(\rho)$. The integration constants are suppressed, since they correspond to point symmetries $\rho \rightarrow b_{1} \rho+b_{0}$ of the Laplace equation (55) and as such they are inessential.

Moreover, $r$ is non-constant, since otherwise $C_{\rho}=8 r_{x} r_{y} / r^{2} q=0$, which we excluded at the beginning of the proof. Then, $\rho$ is non-constant as well and we are free to choose coordinates $x, y$ in such a way that $\rho=x$. Otherwise said, we are free to assume that $r=r(x)$ is given by

$$
x=\int \frac{d r}{r^{3 / 2}+c} .
$$

Now, the above expression for $q$ evaluates to

$$
q=-\frac{3}{4} \frac{r^{3 / 2}+c}{4 \Lambda}
$$

To solve equations (53), we choose

$$
\begin{aligned}
& f_{1}^{1}=0, \quad f_{2}^{1}=\mp \frac{a_{2}}{2 \Lambda r^{3 / 2}}, \\
& f_{1}^{2}=0, \quad f_{2}^{2}= \pm \frac{a_{1}}{2 \Lambda r^{3 / 2}} .
\end{aligned}
$$

Next steps differ according to whether $c=0$ or not.

Assume that $c \neq 0$. With $\rho$ being an arbitrary harmonic function, the equation for $s$ becomes

$$
s_{x x}+s_{y y}-2 \frac{s_{x}^{2}+s_{y}^{2}}{s}+\frac{\left(r^{\frac{3}{2}}+c\right)\left(s_{x} \rho_{x}+s_{y} \rho_{y}\right)}{r}-\frac{3}{4} a_{1}^{2} \frac{\left(r^{2}+c \sqrt{r}\right) s^{2}\left(\rho_{x}^{2}+\rho_{y}^{2}\right)}{\Lambda r^{4}}=0,
$$


which is linearizable in terms of the variable

$$
S=\frac{1}{s}+\frac{a_{1}^{2}}{3 \Lambda c r^{3 / 2}}, \quad \text { i.e., } s=\left(S-\frac{a_{1}^{2}}{3 \Lambda c r^{3 / 2}}\right)^{-1},
$$

giving

$$
S_{x x}+S_{y y}+\frac{r^{3 / 2}+c}{r}\left(\rho_{x} S_{x}+\rho_{y} S_{y}\right)=0 .
$$

With $\rho=x$, Eq. (58) simplifies to

$$
S_{x x}+S_{y y}+\frac{r^{3 / 2}+c}{r} S_{x}=0
$$

and the metric becomes

$$
-\frac{3}{4} \frac{r^{3 / 2}+c}{\sqrt{r} \Lambda}\left(d x^{2}+d y^{2}\right)-2 \frac{d y d u}{\sqrt{r} \Lambda}-2 r d u d v+\frac{4}{3} \frac{\psi r^{2}-\sqrt{r}}{\Lambda c r} d u^{2} .
$$

Choosing $r, y, u, v$ as coordinates, we get the solution (43) and Eq. (44) for the unknown function $\psi$.

Finally, assume that $c=0$. Then, $x=-2 / \sqrt{r}$, so that $r=4 / x^{2}$ and easy computation gives the metric (45).

Remark 5 Let us remark that not only the cylindrical Laplace equation, but also the linear equation (44) is separable by the substitution $\psi(r, y)=R(r) Y(y)$. The $y$-dependent factor $Y(y)$ is easy to find from $Y^{\prime \prime}=\frac{1}{3} \Lambda C Y / c^{2}$ where $C$ is an arbitrary constant. The difficult part is the equation for $R(r)$,

$$
\frac{\left(c^{2} r^{3}+1\right)^{2}}{r^{2}} R_{r r}+\frac{\left(c^{2} r^{3}+1\right)\left(4 c^{2} r^{3}+1\right)}{r^{3}} R_{r}-C R=0 .
$$

It is easily solvable for $C=0$, but to apply the linear superposition principle for solutions we need enough solutions for $C \neq 0$, too. Since $c \neq 0$ by assumption, we can set it to 1 by substitution $r \rightarrow r / c^{2 / 3}$, obtaining

$$
R^{\prime \prime}+\frac{4 r^{3}+1}{r\left(r^{3}+1\right)} R^{\prime}-\frac{C r^{2}}{\left(r^{3}+1\right)^{2}} R=0 .
$$

Equation (61) has five regular singular points given by $r=0, r^{3}+1=0, r=\infty$; at these points it is amenable to convergent series solution.

\section{Differential invariants for $\Lambda$-vacuum Einstein metrics}

In this section we answer the question of how many invariants are functionally independent on solutions of the $\Lambda$-vacuum Einstein equations. Recall that every system of partial differential equations induces a proper subset $\mathcal{E}^{(k)}$ in each $k$ th-order jet space, where $k$ is greater or equal to the order of the equation. The 20 invariants given in Proposition 9 can be easily restricted to $\mathcal{E}^{(k)}$. The easiest way to do this is to solve the equations with respect to a suitable set of the highest order variables, and use them as substitutions.

Proposition 16 The ten (semi-)invariants $C_{\rho}, C_{\chi}, Q_{\chi}, Q_{\gamma}, \ell_{\mathcal{C}}, \Theta_{\mathrm{I}}, X C_{\chi}, X Q_{\gamma}, X^{\perp} C_{\chi}$, $X^{\perp} Q_{\gamma}$ constitute a maximal set of scalar differential (semi-)invariants of order $\leq 2$ functionally independent on generic solutions of the $\Lambda$-vacuum Einstein equations. 
Proof The rank of the Jacobian at a generic point of $\mathcal{E}^{(2)}$ is equal to 10 .

The simplest six relations are

$$
\begin{aligned}
& C_{\text {ric }}+\frac{1}{2} C_{\chi} \mp \tilde{\mathbf{g}} \frac{3}{2} \ell_{\mathcal{C}}=0, \\
& C_{\nu} \mp \tilde{\mathbf{g}} \ell_{\mathcal{C}}+4 \Lambda+\frac{1}{2} C_{\rho}=0, \\
& \pm_{\tilde{\mathbf{g}}}\left(X^{\perp} C_{\rho}\right)^{2}+4 Q_{\chi} C_{\rho}^{2}-16\left(Q_{\chi}-Q_{\gamma}\right) C_{\chi} C_{\rho}+64\left(Q_{\chi}-Q_{\gamma}\right)^{2}=0, \\
& \pm_{\tilde{\mathbf{g}}}\left(X^{\perp} \ell_{\mathcal{C}}\right)^{2} \pm_{\tilde{\mathbf{g}} \mathbf{h}} 4\left(\Theta_{\mathrm{I}}-2 \ell_{\mathcal{C}} \sqrt{Q_{\gamma}}\right) \Theta_{\mathrm{I}}+4 \ell_{\mathcal{C}}^{2} Q_{\chi}=0, \\
& X C_{\rho}+\left(C_{\rho}-C_{\chi}+4 \Lambda \mp \tilde{\mathbf{g}} \ell_{\mathcal{C}}\right) C_{\rho}+8\left(Q_{\chi}-Q_{\gamma}\right)=0, \\
& \left(Q_{\chi}-Q_{\gamma}\right) X \ell_{\mathcal{C}}^{2} \mp \tilde{\mathbf{g} h} C_{\rho} \sqrt{Q_{\gamma}} \Theta_{\mathrm{I}} X \ell_{\mathcal{C}}+\left(3 Q_{\chi}-2 Q_{\gamma}\right) C_{\rho} \ell_{\mathcal{C}} X \ell_{\mathcal{C}} \\
& \quad+\left(C_{\chi} C_{\rho} Q_{\chi}+2 C_{\rho}^{2} Q_{\chi}-C_{\rho}^{2} Q_{\gamma}-4 Q_{\chi}^{2}+4 Q_{\chi} Q_{\gamma}\right) \ell_{\mathcal{C}}^{2} \\
& \quad \mp_{\tilde{\mathbf{g}} \mathbf{h}}\left(2 C_{\chi} C_{\rho}+C_{\rho}^{2}-8 Q_{\chi}\right) \sqrt{Q_{\gamma}} \Theta_{\mathrm{I}} \ell_{\mathcal{C}}-8{\sqrt{Q_{\gamma}}}^{3} \Theta_{\mathrm{I}} \ell_{\mathcal{C}} \\
& \quad \pm_{\tilde{\mathbf{g}} \mathbf{h}}\left(C_{\chi} C_{\rho}-\frac{1}{4} C_{\rho}^{2}-4 Q_{\chi}+4 Q_{\gamma}\right) \Theta_{\mathrm{I}}^{2}=0 .
\end{aligned}
$$

Here $\pm_{\tilde{\mathbf{g}} \mathbf{h}}=\operatorname{sgn}(\operatorname{det} \tilde{\mathbf{g}} \operatorname{det} \mathbf{h}), \mp \tilde{\mathbf{g h}}=-\operatorname{sgn}(\operatorname{det} \tilde{\mathbf{g}} \operatorname{det} \mathbf{h})$ and $\pm_{\tilde{\mathbf{g}}}=\operatorname{sgn}(\operatorname{det} \tilde{\mathbf{g}})$.

Let us turn back to the sectional curvatures (25) and (26).

Proposition 17 The $\Lambda$-vacuum Einstein equations imply that $\Xi$ and $\Xi^{\perp}$ have the same sectional curvatures.

Proof The relation $C_{\text {ric }}+\frac{1}{2} C_{\chi} \mp \tilde{\mathbf{g}} \frac{3}{2} \ell_{\mathcal{C}}=0$ is equivalent to $K\left(\Xi^{\perp}\right)=K(\Xi)$.

\section{The equivalence problem in the case $\ell_{\mathcal{C}} C_{\rho} \neq 0$}

Let $\left\{I_{1}, \ldots, I_{6}\right\}$ be a maximal system of generically functionally independent scalar differential invariants for $\mathfrak{G}^{(1)}$ on $J^{1}(\tau)$. For every metric $\mathbf{g}$, which is a section of $\tau: E \rightarrow \mathcal{M}$, the restrictions $\left.I_{i}\right|_{\mathbf{g}}$ (see Remark 1) to the first-order prolongation of $\mathbf{g}$ provide at most two functionally independent differential invariants on $\mathcal{S}$. The functional relations between the restricted invariants provide necessary conditions for another metric $\overline{\mathbf{g}}$ to be equivalent to $\mathbf{g}$. Here we discuss two different methods for the solution of the equivalence problem, applicable to metrics satisfying $\ell_{\mathcal{C}} C_{\rho} \neq 0$ and possessing at least two functionally independent scalar invariants.

\subsection{The first method}

Let $\mathbf{g}$ and $\overline{\mathbf{g}}$ be two generic metrics which, in adapted coordinates $\left(t^{1}, t^{2}, z^{1}, z^{2}\right)$ and $\left(\bar{t}^{1}, \bar{t}^{2}, \bar{z}^{1}, \bar{z}^{2}\right)$, are written as

$$
\mathbf{g}=b_{i j}\left(t^{1}, t^{2}\right) d t^{i} d t^{j}+2 f_{i k}\left(t^{1}, t^{2}\right) d t^{i} d z^{k}+h_{k l}\left(t^{1}, t^{2}\right) d z^{k} d z^{l},
$$

and

$$
\overline{\mathbf{g}}=\bar{b}_{m n}\left(\bar{t}^{1}, \bar{t}^{2}\right) \mathrm{d} \bar{t}^{m} \mathrm{~d} \bar{t}^{n}+2 \bar{f}_{m r}\left(\bar{t}^{1}, \bar{t}^{2}\right) \mathrm{d} \bar{t}^{m} \mathrm{~d} \bar{z}^{r}+\bar{h}_{r s}\left(\bar{t}^{1}, \bar{t}^{2}\right) \mathrm{d} \bar{z}^{r} \mathrm{~d} \bar{z}^{s},
$$

respectively. If $\mathbf{g}$ and $\overline{\mathbf{g}}$ are equivalent, then there is a pair of indexes $a, b \in\{1,2, \ldots, 6\}$ such that $\left\{\left.I_{a}\right|_{\mathbf{g}}\left(t^{1}, t^{2}\right),\left.I_{b}\right|_{\mathbf{g}}\left(t^{1}, t^{2}\right)\right\}$ and $\left\{\left.\bar{I}_{a}\right|_{\mathbf{g}}\left(\bar{t}^{1}, \bar{t}^{2}\right),\left.\bar{I}_{b}\right|_{\mathbf{g}}\left(\bar{t}^{1}, \bar{t}^{2}\right)\right\}$ are two systems of functionally independent invariants on $\mathcal{S}$. For ease of notation, we will denote by $\left\{\mathcal{I}^{1}\left(t^{1}, t^{2}\right), \mathcal{I}^{2}\left(t^{1}, t^{2}\right)\right\}$ and $\left\{\overline{\mathcal{I}}^{1}\left(\bar{t}^{1}, \bar{t}^{2}\right), \overline{\mathcal{I}}^{2}\left(\bar{t}^{1}, \bar{t}^{2}\right)\right\}$, respectively, these two systems. 
Then, by implicit function theorem, the system

$$
\mathcal{I}^{1}\left(t^{1}, t^{2}\right)=\overline{\mathcal{I}}^{1}\left(\bar{t}^{1}, \bar{t}^{2}\right), \quad \mathcal{I}^{2}\left(t^{1}, t^{2}\right)=\overline{\mathcal{I}}^{2}\left(\bar{t}^{1}, \bar{t}^{2}\right)
$$

locally defines the $\left(t^{1}, t^{2}\right)$-part of coordinate transformation (2), i.e.,

$$
\bar{t}^{i}=\phi^{i}\left(t^{1}, t^{2}\right), \quad i=1,2 .
$$

On the other hand, under a coordinate transformation $P$ of the form (2), the coordinate vector fields transform as

$$
P_{*}\left(\partial_{z^{j}}\right)=\alpha_{j}^{i} \partial_{\bar{z}^{i}}, \quad P_{*}\left(\partial_{t^{j}}\right)=\left(\frac{\partial \psi^{i}}{\partial t^{j}} \circ P^{-1}\right) \partial_{\bar{z}^{i}}+\left(\frac{\partial \phi^{i}}{\partial t^{j}} \circ P^{-1}\right) \partial_{\bar{t}^{i}} .
$$

Hence, in view of relations (see Proposition 7)

$$
P_{*}(\mathcal{C})=\epsilon_{1} \overline{\mathcal{C}}, \quad P_{*}\left(\mathcal{C}^{\perp}\right)=\epsilon_{1} \epsilon_{2} \overline{\mathcal{C}}^{\perp},
$$

where $\epsilon_{1}=\operatorname{sgn}\left(J_{\phi}\right)$ and $\epsilon_{2}=\operatorname{sgn}\left(\operatorname{det}\left(\alpha_{j}^{i}\right)\right)$, one readily gets that

$$
\left(\begin{array}{ll}
\alpha_{1}^{1} & \alpha_{2}^{1} \\
\alpha_{1}^{2} & \alpha_{2}^{2}
\end{array}\right)=\epsilon_{1}\left(\begin{array}{ll}
\overline{\mathcal{C}}^{1} & \epsilon_{2}\left(\overline{\mathcal{C}}^{\perp}\right)^{1} \\
\overline{\mathcal{C}}^{2} & \epsilon_{2}\left(\overline{\mathcal{C}}^{\perp}\right)^{2}
\end{array}\right)\left(\begin{array}{ll}
\mathcal{C}^{1} & \left(\mathcal{C}^{\perp}\right)^{1} \\
\mathcal{C}^{2} & \left(\mathcal{C}^{\perp}\right)^{2}
\end{array}\right)^{-1}
$$

where $\bar{t}^{i}=\phi^{i}\left(t^{1}, t^{2}\right), i=1,2$.

Analogously, in view of relations

$$
P_{*}(\mathcal{H})=\overline{\mathcal{H}}, \quad P_{*}\left(\mathcal{H}^{\perp}\right)=\epsilon_{1} \overline{\mathcal{H}}^{\perp},
$$

one also gets under substitution (2) that

$$
\left(\begin{array}{ll}
\frac{\partial \phi^{1}}{\partial t^{1}} & \frac{\partial \phi^{1}}{\partial t^{2}} \\
\frac{\partial \phi^{2}}{\partial t^{1}} & \frac{\partial \phi^{2}}{\partial t^{2}}
\end{array}\right)=\left(\begin{array}{lll}
\overline{\mathcal{H}}^{1} & \epsilon_{1} & \left.\overline{\mathcal{H}}^{\perp}\right)^{1} \\
\overline{\mathcal{H}}^{2} & \epsilon_{1} & \left(\overline{\mathcal{H}}^{\perp}\right)^{2}
\end{array}\right)\left(\begin{array}{ll}
\mathcal{H}^{1} & \left(\mathcal{H}^{\perp}\right)^{1} \\
\mathcal{H}^{2} & \left(\mathcal{H}^{\perp}\right)^{2}
\end{array}\right)^{-1}
$$

and

$$
\begin{aligned}
\left(\begin{array}{ll}
\frac{\partial \psi^{1}}{\partial t^{1}} & \frac{\partial \psi^{1}}{\partial t^{2}} \\
\frac{\partial \psi^{2}}{\partial t^{1}} & \frac{\partial \psi^{2}}{\partial t^{2}}
\end{array}\right)= & \left(\begin{array}{ll}
\alpha_{1}^{1} & \alpha_{2}^{1} \\
\alpha_{1}^{2} & \alpha_{2}^{2}
\end{array}\right)\left(\begin{array}{ll}
f_{1}^{1} & f_{2}^{1} \\
f_{1}^{2} & f_{2}^{2}
\end{array}\right) \\
& -\left(\begin{array}{cc}
\bar{f}_{1}^{1} & \bar{f}_{2}^{1} \\
\bar{f}_{1}^{2} & \bar{f}_{2}^{2}
\end{array}\right)\left(\begin{array}{ll}
\overline{\mathcal{H}}^{1} & \epsilon_{1}\left(\overline{\mathcal{H}}^{\perp}\right)^{1} \\
\overline{\mathcal{H}}^{2} & \epsilon_{1}\left(\overline{\mathcal{H}}^{\perp}\right)^{2}
\end{array}\right)\left(\begin{array}{ll}
\mathcal{H}^{1} & \left(\mathcal{H}^{\perp}\right)^{1} \\
\mathcal{H}^{2} & \left(\mathcal{H}^{\perp}\right)^{2}
\end{array}\right)^{-1},
\end{aligned}
$$

where $f_{j}^{k}=f_{j s} h^{s k}$ and $\bar{f}_{j}^{k}=\bar{f}_{j s} \bar{h}^{s k}$ with $h^{s k}$ and $\bar{h}^{s k}$ denoting the elements of the inverse matrix $\left(h_{i j}\right)^{-1}$ and $\left(\bar{h}_{i j}\right)^{-1}$, respectively.

Thus, one gets the following theorem:

Theorem 2 The metrics

$$
\mathbf{g}=b_{i j}\left(t^{1}, t^{2}\right) d t^{i} d t^{j}+2 f_{i k}\left(t^{1}, t^{2}\right) d t^{i} d z^{k}+h_{k l}\left(t^{1}, t^{2}\right) d z^{k} d z^{l}
$$

and

$$
\overline{\mathbf{g}}=\bar{b}_{m n}\left(\bar{t}^{1}, \bar{t}^{2}\right) \mathrm{d} \bar{t}^{m} \mathrm{~d} \bar{t}^{n}+2 \bar{f}_{m r}\left(\bar{t}^{1}, \bar{t}^{2}\right) \mathrm{d} \bar{t}^{m} \mathrm{~d} \bar{z}^{r}+\bar{h}_{r s}\left(\bar{t}^{1}, \bar{t}^{2}\right) \mathrm{d} \bar{z}^{r} \mathrm{~d} \bar{z}^{s},
$$


with $\ell_{\mathcal{C}} C_{\rho} \neq 0$ and $\bar{\ell}_{\overline{\mathcal{C}}} \bar{C}_{\bar{\rho}} \neq 0$, respectively, are equivalent if and only if there exists a coordinate transformation

$$
P: \quad \bar{t}^{i}=\phi^{i}\left(t^{1}, t^{2}\right), \quad \bar{z}^{i}=\alpha_{j}^{i} z^{j}+\psi^{i}\left(t^{1}, t^{2}\right), \quad\left(\alpha_{j}^{i}\right) \in \mathrm{GL}(2, \mathbb{R})
$$

satisfying the following conditions:

(i) $\left\{\mathcal{I}^{1}=\left.I_{a}\right|_{\mathbf{g}}, \mathcal{I}^{2}=\left.I_{b}\right|_{\mathbf{g}}\right\}$ and $\left\{\overline{\mathcal{I}}^{1}=\left.\bar{I}_{a}\right|_{\overline{\mathbf{g}}}, \overline{\mathcal{I}}^{2}=\left.\bar{I}_{b}\right|_{\mathbf{g}}\right\}$, for some $a, b \in\{1,2, \ldots, 6\}$, are two systems of functionally independent scalar invariants on $\mathcal{S}$ and the coordinate transformation $\left\{\bar{t}^{1}=\phi^{1}\left(t^{1}, t^{2}\right), \bar{t}^{2}=\phi^{2}\left(t^{1}, t^{2}\right)\right\}$ is implicitly defined by

$$
\mathcal{I}^{1}-\overline{\mathcal{I}}^{1}=0, \quad \mathcal{I}^{2}-\overline{\mathcal{I}}^{2}=0
$$

(ii) the right-hand side of (65), with $\epsilon_{1}=\operatorname{sgn}\left(J_{\phi}\right)$ and $\epsilon_{2}=\operatorname{sgn}\left(\operatorname{det}\left(\alpha_{j}^{i}\right)\right)$, is a constant matrix coinciding with $\left(\alpha_{j}^{i}\right) \in \mathrm{GL}(2, \mathbb{R})$;

(iii) the transformation $\left\{\bar{t}^{1}=\phi^{1}\left(t^{1}, t^{2}\right), \bar{t}^{2}=\phi^{2}\left(t^{1}, t^{2}\right)\right\}$, defined in (i), satisfies (66);

(iv) the functions $\psi^{i}=\psi^{i}\left(t^{1}, t^{2}\right), i=1,2$, are solutions of an integrable system of firstorder partial differential equations defined by (67);

(v) the matrix $\left(\alpha_{j}^{i}\right)$ and the derivatives of $\phi^{i}$ and $\psi^{i}$ satisfy the system (7) for $\mathbf{g}$ and $\overline{\mathbf{g}}$.

\subsection{The second method}

Let $\mathbf{g}$ and $\mathbf{g}$ be two metrics which, in adapted coordinates $\left(t^{1}, t^{2}, z^{1}, z^{2}\right)$ and $\left(\bar{t}^{1}, \bar{t}^{2}, \bar{z}^{1}, \bar{z}^{2}\right)$, read as (62) and (63), respectively. Under the assumption $\ell_{\mathcal{C}} C_{\rho} \neq 0$, by using the semiinvariant orthogonal frames (see Proposition 7)

$$
\left\{\mathcal{Y}_{1}=\mathcal{H}, \mathcal{Y}_{2}=\mathcal{H}^{\perp}, \mathcal{Y}_{3}=\mathcal{C}, \mathcal{Y}_{4}=\mathcal{C}^{\perp}\right\}
$$

and

$$
\left\{\overline{\mathcal{Y}}_{1}=\overline{\mathcal{H}}, \overline{\mathcal{Y}}_{2}=\overline{\mathcal{H}}^{\perp}, \overline{\mathcal{Y}}_{3}=\overline{\mathcal{C}}, \overline{\mathcal{Y}}_{4}=\overline{\mathcal{C}}^{\perp}\right\},
$$

and the corresponding semi-invariant dual co-frames $\left\{\omega_{1}, \omega_{2}, \omega_{3}, \omega_{4}\right\}$ and $\left\{\bar{\omega}_{1}, \bar{\omega}_{2}, \bar{\omega}_{3}, \bar{\omega}_{4}\right\}$, $\mathbf{g}$ and $\overline{\mathbf{g}}$ can be written as

$$
\mathbf{g}=\ell_{\mathcal{H}} \omega_{1}^{2}+\ell_{\mathcal{H}^{\perp}} \omega_{2}^{2}+\ell_{\mathcal{C}} \omega_{3}^{2}+\ell_{\mathcal{C}^{\perp}} \omega_{4}^{2}
$$

and

$$
\overline{\mathbf{g}}=\bar{\ell}_{\overline{\mathcal{H}}} \bar{\omega}_{1}^{2}+\bar{\ell}_{\overline{\mathcal{H}}^{\perp}} \bar{\omega}_{2}^{2}+\bar{\ell}_{\overline{\mathcal{C}}} \bar{\omega}_{3}^{2}+\bar{\ell}_{\overline{\mathcal{C}}^{\perp}} \bar{\omega}_{4}^{2},
$$

respectively.

We notice that in view of (23) the co-frame transforms as

$$
\omega_{1} \mapsto \omega_{1}, \quad \omega_{2} \mapsto\left(\operatorname{sgn} J_{\phi}\right) \omega_{2}, \quad \omega_{3} \mapsto\left(\operatorname{sgn} J_{\phi}\right) \omega_{3}, \quad \omega_{4} \mapsto\left(\operatorname{sgn} J_{\phi}\right)\left(\operatorname{sgn} \operatorname{det} \alpha_{j}^{i}\right) \omega_{4}(68)
$$

under the pseudogroup action (2). Moreover, in terms of local adapted coordinates $\left\{t^{1}, t^{2}, z^{1}, z^{2}\right\}$, one has

$$
\left(\begin{array}{l}
\omega_{1} \\
\omega_{2}
\end{array}\right)=\left(\begin{array}{ll}
\mathcal{H}^{1} & \left(\mathcal{H}^{\perp}\right)^{1} \\
\mathcal{H}^{2} & \left(\mathcal{H}^{\perp}\right)^{2}
\end{array}\right)^{-1}\left(\begin{array}{l}
d t^{1} \\
d t^{2}
\end{array}\right)
$$


and

$$
\left(\begin{array}{l}
\omega_{3} \\
\omega_{4}
\end{array}\right)=\left(\begin{array}{ll}
\mathcal{C}^{1} & \left(\mathcal{C}^{\perp}\right)^{1} \\
\mathcal{C}^{2} & \left(\mathcal{C}^{\perp}\right)^{2}
\end{array}\right)^{-1}\left[\left(\begin{array}{ll}
f_{1}^{1} & f_{2}^{1} \\
f_{1}^{2} & f_{2}^{2}
\end{array}\right)\left(\begin{array}{l}
d t^{1} \\
d t^{2}
\end{array}\right)+\left(\begin{array}{l}
d z^{1} \\
d z^{2}
\end{array}\right)\right] .
$$

From now on, we assume that there is a pair of indexes $a, b \in\{1,2, \ldots, 6\}$ such that $\left\{\left.I_{a}\right|_{\mathbf{g}}\left(t^{1}, t^{2}\right),\left.I_{b}\right|_{\mathbf{g}}\left(t^{1}, t^{2}\right)\right\}$ and $\left\{\left.\bar{I}_{a}\right|_{\mathbf{g}}\left(\bar{t}^{1}, \bar{t}^{2}\right), \bar{I}_{b} \mid \overline{\mathbf{g}}_{(}\left(\bar{t}^{1}, \bar{t}^{2}\right)\right\}$ are two systems of functionally independent invariants on $\mathcal{S}$. For ease of notation, we will denote these two systems by $\left\{\mathcal{I}^{1}\left(t^{1}, t^{2}\right), \mathcal{I}^{2}\left(t^{1}, t^{2}\right)\right\}$ and $\left\{\overline{\mathcal{I}}^{1}\left(\bar{t}^{1}, \bar{t}^{2}\right), \overline{\mathcal{I}}^{2}\left(\bar{t}^{1}, \bar{t}^{2}\right)\right\}$, respectively. In view of the functional independence, $\left\{\mathcal{I}^{1}, \mathcal{I}^{2}, z^{1}, z^{2}\right\}$ and $\left\{\overline{\mathcal{I}}^{1}, \overline{\mathcal{I}}^{2}, \bar{z}^{1}, \bar{z}^{2}\right\}$ define new adapted coordinates for $\mathbf{g}$ and $\overline{\mathbf{g}}$, respectively. Therefore, by the implicit function theorem, $\left\{\mathcal{I}^{1}=\mathcal{I}^{1}\left(t^{1}, t^{2}\right), \mathcal{I}^{2}=\mathcal{I}^{2}\left(t^{1}, t^{2}\right)\right\}$ and $\left\{\overline{\mathcal{I}}^{1}=\overline{\mathcal{I}}^{1}\left(\bar{t}^{1}, \bar{t}^{2}\right), \overline{\mathcal{I}}^{2}=\overline{\mathcal{I}}^{2}\left(\bar{t}^{1}, \bar{t}^{2}\right)\right\}$ define local coordinate transformations

$$
t^{i}=m^{i}\left(\mathcal{I}^{1}, \mathcal{I}^{2}\right), \quad i=1,2
$$

and

$$
\bar{t}^{i}=\bar{m}^{i}\left(\overline{\mathcal{I}}^{1}, \overline{\mathcal{I}}^{2}\right), \quad i=1,2 .
$$

Then, according to (69) and (70), $\left\{\omega_{1}, \omega_{2}, \omega_{3}, \omega_{4}\right\}$ can be written in terms of new adapted coordinates $\left\{\mathcal{I}^{1}, \mathcal{I}^{2}, z^{1}, z^{2}\right\}$ as

$$
\omega_{h}=a_{h i} \mathrm{~d} \mathcal{I}^{i}+p_{h i} d z^{i},
$$

where $a_{h i}=a_{h i}\left(\mathcal{I}^{1}, \mathcal{I}^{2}\right), p_{h i}=p_{h i}\left(\mathcal{I}^{1}, \mathcal{I}^{2}\right)$ and

$$
\operatorname{det}\left(\begin{array}{ll}
a_{11} & a_{12} \\
a_{21} & a_{22}
\end{array}\right) \neq 0, \quad \operatorname{det}\left(\begin{array}{ll}
p_{31} & p_{32} \\
p_{41} & p_{42}
\end{array}\right) \neq 0, \quad p_{1 i}=p_{2 i}=0 .
$$

In particular, the coefficients $a_{h i}$ and $p_{h i}$ can be computed by using the identities

$$
\left(\begin{array}{c}
\omega_{1} \\
\omega_{2}
\end{array}\right)=\left(\begin{array}{ll}
\mathcal{H}^{1} & \left(\mathcal{H}^{\perp}\right)^{1} \\
\mathcal{H}^{2} & \left(\mathcal{H}^{\perp}\right)^{2}
\end{array}\right)_{\text {invar. }}^{-1}\left(\begin{array}{ll}
\frac{\partial m^{1}}{\partial \mathcal{I}^{1}} & \frac{\partial m^{1}}{\partial \mathcal{I}^{2}} \\
\frac{\partial m^{2}}{\partial \mathcal{I}^{1}} & \frac{\partial m^{2}}{\partial \mathcal{I}^{2}}
\end{array}\right)\left(\begin{array}{l}
\mathrm{d} \mathcal{I}^{1} \\
\mathrm{~d} \mathcal{I}^{2}
\end{array}\right)
$$

and

$$
\left(\begin{array}{l}
\omega_{3} \\
\omega_{4}
\end{array}\right)=\left(\begin{array}{cc}
\mathcal{C}^{1} & \left(\mathcal{C}^{\perp}\right)^{1} \\
\mathcal{C}^{2} & \left(\mathcal{C}^{\perp}\right)^{2}
\end{array}\right)_{\text {invar. }}^{-1}\left[\left(\begin{array}{ll}
f_{1}^{1} & f_{2}^{1} \\
f_{1}^{2} & f_{2}^{2}
\end{array}\right)_{\text {invar. }}\left(\begin{array}{ll}
\frac{\partial m^{1}}{\partial \mathcal{I}^{1}} & \frac{\partial m^{1}}{\partial \mathcal{I}^{2}} \\
\frac{\partial m^{2}}{\partial \mathcal{I}^{1}} & \frac{\partial m^{2}}{\partial \mathcal{I}^{2}}
\end{array}\right)\left(\begin{array}{l}
\mathrm{d} \mathcal{I}^{1} \\
\mathrm{~d} \mathcal{I}^{2}
\end{array}\right)+\left(\begin{array}{l}
d z^{1} \\
d z^{2}
\end{array}\right)\right]
$$

where "invar." means the restriction to $\left\{t^{i}=m^{i}\left(\mathcal{I}^{1}, \mathcal{I}^{2}\right)\right\}$.

Analogously, one can write $\left\{\bar{\omega}_{1}, \bar{\omega}_{2}, \bar{\omega}_{3}, \bar{\omega}_{4}\right\}$ in terms of the adapted coordinates $\left\{\overline{\mathcal{I}}^{1}, \overline{\mathcal{I}}^{2}, \bar{z}^{1}, \bar{z}^{2}\right\}$ as

$$
\bar{\omega}_{i}=\bar{a}_{h i} \mathrm{~d} \overline{\mathcal{I}}^{i}+\bar{p}_{h i} \mathrm{~d} \bar{z}^{i},
$$

where the coefficients $\bar{a}_{h i}=\bar{a}_{h i}\left(\overline{\mathcal{I}}^{1}, \overline{\mathcal{I}}^{2}\right), \bar{p}_{h i}=\bar{p}_{h i}\left(\overline{\mathcal{I}}^{1}, \overline{\mathcal{I}}^{2}\right)$ are such that

$$
\operatorname{det}\left(\begin{array}{ll}
\bar{a}_{11} & \bar{a}_{12} \\
\bar{a}_{21} & \bar{a}_{22}
\end{array}\right) \neq 0, \quad \operatorname{det}\left(\begin{array}{ll}
\bar{p}_{31} & \bar{p}_{32} \\
\bar{p}_{41} & \bar{p}_{42}
\end{array}\right) \neq 0, \quad \bar{p}_{1 i}=\bar{p}_{2 i}=0
$$

and can be computed by using formulas analogous to (71) and (72) (where in this case "invar." means the restriction to $\left.\left\{\bar{t}^{i}=\bar{m}^{i}\left(\overline{\mathcal{I}}^{1}, \overline{\mathcal{I}}^{2}\right)\right\}\right)$. 
Lemma 5 Under the pseudogroup action (2), the coefficients $a_{i j}=\omega_{i}\left(\partial_{\mathcal{I}^{j}}\right)$ transform according to the following formulas

$$
\bar{a}_{1 i}=a_{1 i}, \quad \bar{a}_{2 i}=\epsilon_{1} a_{2 i}, \quad \bar{a}_{3 i}=\epsilon_{1} a_{3 i}, \quad \bar{a}_{4 i}=\epsilon_{1} \epsilon_{2} a_{4 i},
$$

with $\epsilon_{1}=\operatorname{sgn} J_{\phi}$ and $\epsilon_{2}=\operatorname{sgn} \operatorname{det} \alpha_{j}^{i}$, whereas the coefficients $p_{h i}=\omega_{h}\left(\partial_{z^{i}}\right)$ transform as

$$
p_{3 i}=\epsilon_{1} \bar{p}_{3 s} \alpha_{i}^{s}, \quad p_{4 i}=\epsilon_{1} \epsilon_{2} \bar{p}_{4 s} \alpha_{i}^{s},
$$

with $\left(\alpha_{j}^{i}\right) \in \mathrm{GL}(2, \mathbb{R})$ such that

$$
\left(\begin{array}{ll}
\alpha_{1}^{1} & \alpha_{2}^{1} \\
\alpha_{1}^{2} & \alpha_{2}^{2}
\end{array}\right)=\epsilon_{1}\left(\begin{array}{ll}
\overline{\mathcal{C}}^{1} & \epsilon_{2}\left(\overline{\mathcal{C}}^{\perp}\right)^{1} \\
\overline{\mathcal{C}}^{2} & \epsilon_{2}\left(\overline{\mathcal{C}}^{\perp}\right)^{2}
\end{array}\right)_{\text {invar. }}\left(\begin{array}{ll}
\mathcal{C}^{1} & \left(\mathcal{C}^{\perp}\right)^{1} \\
\mathcal{C}^{2} & \left(\mathcal{C}^{\perp}\right)^{2}
\end{array}\right)_{\text {invar. }}^{-1} .
$$

Proof Equations (73) and (74) readily follow by (68). On the other hand, when $\bar{\omega}_{3}, \bar{\omega}_{4}$ are obtained by $\omega_{3}, \omega_{4}$ through the pseudogroup action (2), equations (73) and (74) entail (75).

Notice that (75) is the same condition (65) obtained in the first method. One also has the following lemma.

Lemma 6 The fact that right-hand side of (75) is an element of $\operatorname{GL}(2, \mathbb{R})$ is equivalent to the following condition

$$
\begin{aligned}
& \left(\begin{array}{ll}
\overline{\mathcal{C}}^{1} \epsilon_{2}\left(\overline{\mathcal{C}}^{\perp}\right)^{1} \\
\overline{\mathcal{C}}^{2} \epsilon_{2}\left(\overline{\mathcal{C}}^{\perp}\right)^{2}
\end{array}\right)_{\text {invar. }}^{-1} \frac{\partial}{\partial \mathcal{I}^{s}}\left(\begin{array}{l}
\overline{\mathcal{C}}^{1} \epsilon_{2}\left(\overline{\mathcal{C}}^{\perp}\right)^{1} \\
\overline{\mathcal{C}}^{2} \epsilon_{2}\left(\overline{\mathcal{C}}^{\perp}\right)^{2}
\end{array}\right)_{\text {invar. }} \\
& =\left(\begin{array}{l}
\mathcal{C}^{1}\left(\mathcal{C}^{\perp}\right)^{1} \\
\mathcal{C}^{2}\left(\mathcal{C}^{\perp}\right)^{2}
\end{array}\right)_{\text {invar. }}^{-1} \frac{\partial}{\partial \mathcal{I}^{s}}\left(\begin{array}{l}
\mathcal{C}^{1}\left(\mathcal{C}^{\perp}\right)^{1} \\
\mathcal{C}^{2}\left(\mathcal{C}^{\perp}\right)^{2}
\end{array}\right)_{\text {invar. }}, \quad s=1,2 .
\end{aligned}
$$

In particular, under transformations preserving the orientations of the leaves of $\Xi$, (76) is equivalent to the invariance of the matrices

$$
\left(\begin{array}{ll}
\mathcal{C}^{1} & \left(\mathcal{C}^{\perp}\right)^{1} \\
\mathcal{C}^{2} & \left(\mathcal{C}^{\perp}\right)^{2}
\end{array}\right)_{\text {invar. }}^{-1} \frac{\partial}{\partial \mathcal{I}^{s}}\left(\begin{array}{ll}
\mathcal{C}^{1} & \left(\mathcal{C}^{\perp}\right)^{1} \\
\mathcal{C}^{2} & \left(\mathcal{C}^{\perp}\right)^{2}
\end{array}\right)_{\text {invar. }} \quad, \quad s=1,2 .
$$

Moreover, by introducing the functions

$$
\begin{gathered}
\mathfrak{c}_{11}^{s}=\frac{\left(\mathcal{C}^{\perp}\right)^{2} \frac{\partial \mathcal{C}^{1}}{\partial \mathcal{I}^{s}}-\left(\mathcal{C}^{\perp}\right)^{1} \frac{\partial \mathcal{C}^{2}}{\partial \mathcal{I}^{s}}}{\mathcal{C}^{1}\left(\mathcal{C}^{\perp}\right)^{2}-\mathcal{C}^{2}\left(\mathcal{C}^{\perp}\right)^{1}}, \quad \mathfrak{c}_{22}^{s}=\frac{\mathcal{C}^{1} \frac{\partial\left(\mathcal{C}^{\perp}\right)^{2}}{\partial \mathcal{I}^{s}}-\mathcal{C}^{2} \frac{\partial\left(\mathcal{C}^{\perp}\right)^{1}}{\partial \mathcal{I}^{s}}}{\mathcal{C}^{1}\left(\mathcal{C}^{\perp}\right)^{2}-\mathcal{C}^{2}\left(\mathcal{C}^{\perp}\right)^{1}} \\
\mathfrak{c}_{12}^{s}=\frac{\left(\mathcal{C}^{\perp}\right)^{2} \frac{\partial\left(\mathcal{C}^{\perp}\right)^{1}}{\partial \mathcal{I}^{s}}-\left(\mathcal{C}^{\perp}\right)^{1} \frac{\partial\left(\mathcal{C}^{\perp}\right)^{2}}{\partial \mathcal{I}^{s}}}{\mathcal{C}^{1}\left(\mathcal{C}^{\perp}\right)^{2}-\mathcal{C}^{2}\left(\mathcal{C}^{\perp}\right)^{1}}, \quad \mathfrak{c}_{21}^{s}=\frac{\mathcal{C}^{1} \frac{\partial \mathcal{C}^{2}}{\partial \mathcal{I}^{s}}-\mathcal{C}^{2} \frac{\partial \mathcal{C}^{1}}{\partial \mathcal{I}^{s}}}{\mathcal{C}^{1}\left(\mathcal{C}^{\perp}\right)^{2}-\mathcal{C}^{2}\left(\mathcal{C}^{\perp}\right)^{1}} .
\end{gathered}
$$

where $s=1,2$, condition (76) is equivalent to the identities

$$
\mathfrak{c}_{11}^{s}=\overline{\mathfrak{c}}_{11}^{s}, \quad \mathfrak{c}_{22}^{s}=\overline{\mathfrak{c}}_{22}^{s}, \quad \mathfrak{c}_{12}^{s}=\epsilon_{2} \overline{\mathfrak{c}}_{12}^{s}, \quad \mathfrak{c}_{21}^{s}=\epsilon_{2} \overline{\mathfrak{c}}_{21}^{s},
$$

Proof By differentiating (75) with respect to $\mathcal{I}^{s}$ and observing that $\mathcal{I}^{s}=\overline{\mathcal{I}}^{s}$, one gets (76). Conversely, (75) follows by integrating (76). The rest of the proof follows by straightforward computations. 
Now the following remark is in order.

Remark 6 In view of (75), transformation formulas (74) are equivalent to

$$
\mathfrak{p}_{3}=\epsilon_{1} \overline{\mathfrak{p}}_{3}, \quad \mathfrak{p}_{4}=\epsilon_{2} \overline{\mathfrak{p}}_{4}, \quad \mathfrak{p}_{3}^{\perp}=\epsilon_{1} \epsilon_{2} \overline{\mathfrak{p}}_{3}^{\perp}, \quad \mathfrak{p}_{4}^{\perp}=\overline{\mathfrak{p}}_{4}^{\perp},
$$

where $\mathfrak{p}_{a}=p_{a s} \mathcal{C}^{s}, \mathfrak{p}_{a}^{\perp}=p_{a s}\left(\mathcal{C}^{\perp}\right)^{s}, a=3,4$. On the other hand, (76) is equivalent to the identities (78). Thus, (73), (74) and (75) are equivalent to the invariance of the 18 functions

$$
\begin{aligned}
& a_{1 i}, \quad \mathfrak{p}_{4}^{\perp}, \quad\left(a_{2 i}\right)^{2},\left(a_{3 i}\right)^{2},\left(a_{4 i}\right)^{2}, a_{2 i} a_{3 i}, \quad\left(\mathfrak{p}_{3}\right)^{2}, \\
& \left(\mathfrak{p}_{3}^{\perp}\right)^{2}, \quad\left(\mathfrak{p}_{4}\right)^{2}, \quad \mathfrak{p}_{3} a_{2 i}, \quad \mathfrak{p}_{3} a_{3 i}, \quad \mathfrak{p}_{3}^{\perp} a_{4 i}, \quad \mathfrak{p}_{3} \mathfrak{p}_{4} a_{4 i}, \mathfrak{p}_{3} \mathfrak{p}_{3}^{\perp} \mathfrak{p}_{4}, \\
& \left(\mathfrak{c}_{12}^{s}\right)^{2},\left(\mathfrak{c}_{21}^{s}\right)^{2}, \mathfrak{c}_{12}^{s} \mathfrak{p}_{4}, \quad \mathfrak{c}_{21}^{s} \mathfrak{p}_{4}
\end{aligned}
$$

with $i, s=1,2$.

\section{Theorem 3 Two metrics}

$$
\mathbf{g}=b_{i j}\left(t^{1}, t^{2}\right) d t^{i} d t^{j}+2 f_{i k}\left(t^{1}, t^{2}\right) d t^{i} d z^{k}+h_{k l}\left(t^{1}, t^{2}\right) d z^{k} d z^{l}
$$

and

$$
\overline{\mathbf{g}}=\bar{b}_{m n}\left(\bar{t}^{1}, \bar{t}^{2}\right) \mathrm{d} \bar{t}^{m} \mathrm{~d} \bar{t}^{n}+2 \bar{f}_{m r}\left(\bar{t}^{1}, \bar{t}^{2}\right) \mathrm{d} \bar{t}^{m} \mathrm{~d} \bar{z}^{r}+\bar{h}_{r s}\left(\bar{t}^{1}, \bar{t}^{2}\right) \mathrm{d} \bar{z}^{r} \mathrm{~d} \bar{z}^{s},
$$

with $\ell_{\mathcal{C}} C_{\rho} \neq 0$ and $\bar{\ell}_{\overline{\mathcal{C}}} \bar{C}_{\bar{\rho}} \neq 0$, respectively, are equivalent if and only if there are two systems $\left\{\mathcal{I}^{1}\left(t^{1}, t^{2}\right), \mathcal{I}^{2}\left(t^{1}, t^{2}\right)\right\}$ and $\left\{\overline{\mathcal{I}}^{1}\left(\bar{t}^{1}, \bar{t}^{2}\right), \overline{\mathcal{I}}^{2}\left(\bar{t}^{1}, \bar{t}^{2}\right)\right\}$ of functionally independent firstorder scalar differential invariants on $\mathcal{S}$, such that the six fundamental first-order scalar differential invariants of $\mathbf{g}$ depend on $\left(\mathcal{I}^{1}, \mathcal{I}^{2}\right)$ in the same way as the corresponding six fundamental invariants of $\overline{\mathbf{g}}$ depend on $\left(\overline{\mathcal{I}}^{1}, \overline{\mathcal{I}}^{2}\right)$.

Proof If $\mathbf{g}$ and $\overline{\mathbf{g}}$ are two equivalent metrics such that $\ell_{\mathcal{C}} C_{\rho} \neq 0$ and $\bar{\ell}_{\overline{\mathcal{C}}} \bar{C}_{\bar{\rho}} \neq 0$, then there are certainly two systems $\left\{\mathcal{I}^{1}\left(t^{1}, t^{2}\right), \mathcal{I}^{2}\left(t^{1}, t^{2}\right)\right\}$ and $\left\{\overline{\mathcal{I}}^{1}\left(\bar{t}^{1}, \bar{t}^{2}\right), \overline{\mathcal{I}}^{2}\left(\bar{t}^{1}, \bar{t}^{2}\right)\right\}$ of functionally independent first-order scalar differential invariants on $\mathcal{S}$ such that the six fundamental first-order scalar differential invariants of $\mathbf{g}$ depend on $\left(\mathcal{I}^{1}, \mathcal{I}^{2}\right)$ in the same way as the corresponding six fundamental invariants of $\overline{\mathbf{g}}$ depend on $\left(\overline{\mathcal{I}}^{1}, \overline{\mathcal{I}}^{2}\right)$.

For the proof of the converse, we first observe that in the generic case, all first-order scalar differential invariants are functions of the six fundamental scalar differential invariants. Hence, when restricting to a metric $\mathbf{g}$ with two functionally independent scalar differential invariants $\left(\mathcal{I}^{1}, \mathcal{I}^{2}\right)$, all first-order scalar differential invariants become functions of $\left(\mathcal{I}^{1}, \mathcal{I}^{2}\right)$. Then, under the given assumptions, all first-order scalar differential invariants of $\mathbf{g}$ depend on $\left(\mathcal{I}^{1}, \mathcal{I}^{2}\right)$ in the same way as all the corresponding first-order scalar differential invariants of $\overline{\mathbf{g}}$ depend on $\left(\overline{\mathcal{I}}^{1}, \overline{\mathcal{I}}^{2}\right)$. Consequently, the invariants (79) for $\mathbf{g}$ transform to the corresponding invariants for $\overline{\mathbf{g}}$ under the transformation $\left\{\overline{\mathcal{I}}^{1}=\mathcal{I}^{1}, \overline{\mathcal{I}}^{2}=\mathcal{I}^{2}\right\}$. Hence, in view of Lemma 5 , Lemma 6 and Remark 6, the matrix $\left(\alpha_{j}^{i}\right)$ defined by (75) belongs to GL(2, R) and defines the adapted coordinate transformation

$$
P: \quad \overline{\mathcal{I}}^{1}=\mathcal{I}^{1}, \quad \overline{\mathcal{I}}^{2}=\mathcal{I}^{2}, \quad \bar{z}^{i}=\alpha_{j}^{i} z^{j}
$$

by which $\left\{\omega_{1}, \omega_{2}, \omega_{3}, \omega_{4}\right\}$ transforms to $\left\{\bar{\omega}_{1}, \bar{\omega}_{2}, \bar{\omega}_{3}, \bar{\omega}_{4}\right\}$. It follows that $P$ is an isometry between $\mathbf{g}$ and $\overline{\mathbf{g}}$ :

$$
\begin{aligned}
P^{*}(\overline{\mathbf{g}}) & =P^{*}\left(\bar{\ell}_{\overline{\mathcal{H}}} \bar{\omega}_{1}^{2}+\bar{\ell}_{\overline{\mathcal{H}}^{\perp}} \bar{\omega}_{2}^{2}+\bar{\ell}_{\overline{\mathcal{C}}} \bar{\omega}_{3}^{2}+\bar{\ell}_{\overline{\mathcal{C}}^{\perp}} \bar{\omega}_{4}^{2}\right) \\
& =\ell_{\mathcal{H}} \omega_{1}^{2}+\ell_{\mathcal{H}^{\perp}} \omega_{2}^{2}+\ell_{\mathcal{C}} \omega_{3}^{2}+\ell_{\mathcal{C}^{\perp}} \omega_{4}^{2}=\mathbf{g} .
\end{aligned}
$$


Corollary 1 The equivalence class of a metric $\mathbf{g}$ such that $\ell_{\mathcal{C}} C_{\rho} \neq 0$ is completely characterized by the way the six first-order scalar differential invariants $I_{1}=C_{\rho}, I_{2}=C_{\chi}, I_{3}=Q_{\chi}$, $I_{4}=Q_{\gamma}, I_{5}=\ell_{\mathcal{C}}, I_{6}=\left(\Theta_{\mathrm{I}}\right)^{2}$ depend on two functionally independent first-order scalar differential invariants $\left(\mathcal{I}^{1}, \mathcal{I}^{2}\right)$.

\subsubsection{Example}

We consider here the Van den Bergh metric

$$
\begin{aligned}
\mathbf{g}= & \cosh \left(\sqrt{6} t^{1}\right)\left\{\sinh ^{4}\left(t^{2}\right)\left[\left(d t^{1}\right)^{2}-\left(d t^{2}\right)^{2}\right]\right. \\
& \left.+2 \sinh ^{2}\left(t^{2}\right)\left[d z^{2}+\cosh \left(t^{2}\right) d t^{1}\right]^{2}\right\} \\
& +\frac{12}{\cosh \left(\sqrt{6} t^{1}\right)}\left[d z^{1}+\cosh \left(t^{2}\right) d z^{2}+\frac{1}{2} \cosh ^{2}\left(t^{2}\right) d t^{1}\right]^{2} .
\end{aligned}
$$

This is a Ricci-flat metric with two Killing vector fields $\partial_{z^{1}}$ and $\partial_{z^{2}}$ and an orthogonally intransitive $\Xi$.

In this case the six fundamental first-order scalar differential invariants are

$$
\begin{aligned}
C_{\rho} & =-4 \frac{\cosh ^{2}\left(t^{2}\right)}{\cosh \left(\sqrt{6} t^{1}\right) \sinh ^{6}\left(t^{2}\right)}, \quad C_{\chi}=-6 \frac{\sinh ^{2}\left(\sqrt{6} t^{1}\right)-1}{\cosh ^{3}\left(\sqrt{6} t^{1}\right) \sinh ^{4}\left(t^{2}\right)}, \\
Q_{\chi} & =6 \frac{\sinh ^{2}\left(\sqrt{6} t^{1}\right)\left[-6 \sinh ^{2}\left(t^{2}\right)+\cosh ^{2}\left(t^{2}\right) \cosh ^{2}\left(\sqrt{6} t^{1}\right)\right]}{\cosh ^{6}\left(\sqrt{6} t^{1}\right) \sinh ^{10}\left(t^{2}\right)} \\
Q_{\gamma} & =-36 \frac{\sinh ^{2}\left(\sqrt{6} t^{1}\right)}{\cosh ^{6}\left(\sqrt{6} t^{1}\right) \sinh ^{8}\left(t^{2}\right)}, \quad \ell_{\mathcal{C}}=2 \frac{1}{\cosh \left(\sqrt{6} t^{1}\right) \sinh ^{4}\left(t^{2}\right)}, \\
\left(\Theta_{1}\right)^{2} & =144 \frac{\sinh ^{2}\left(\sqrt{6} t^{1}\right)}{\sinh ^{16}\left(t^{2}\right) \cosh ^{8}\left(\sqrt{6} t^{1}\right)} .
\end{aligned}
$$

Then, by choosing $\mathcal{I}^{1}=C_{\rho}, \mathcal{I}^{2}=\ell_{\mathcal{C}}$, one can write

$$
t^{1}=\frac{1}{\sqrt{6}} \operatorname{arccosh}\left(\frac{2}{\ell_{\mathcal{C}}}\left(\frac{C_{\rho}}{2 \ell_{\mathcal{C}}}+1\right)^{2}\right), \quad t^{2}=\operatorname{arccoth}\left(\sqrt{1-\frac{1}{2} \frac{C_{\rho}}{\ell_{\mathcal{C}}}}\right) .
$$

It follows that by substituting (81) in (80), the remaining 4 fundamental first-order scalar differential invariants reduce to the following functions of $\mathcal{I}^{1}=C_{\rho}$ and $\mathcal{I}^{2}=\ell_{\mathcal{C}}$ :

$$
\begin{aligned}
C_{\chi} & =\frac{3 \ell_{\mathcal{C}}\left(8 \ell_{\mathcal{C}}^{6}-\left(C_{\rho}^{2}+4 C_{\rho} \ell_{\mathcal{C}}+4 \ell_{\mathcal{C}}^{2}\right)^{2}\right)}{\left(C_{\rho}+2 \ell_{\mathcal{C}}\right)^{4}} \\
Q_{\chi} & =\frac{3 \ell_{\mathcal{C}}\left(48 \ell_{\mathcal{C}}^{7}+C_{\rho}\left(C_{\rho}^{2}+4 C_{\rho} \ell_{\mathcal{C}}+4 \ell_{\mathcal{C}}^{2}\right)^{2}\right)\left(4 \ell_{\mathcal{C}}^{6}-\left(C_{\rho}^{2}+4 C_{\rho} \ell_{\mathcal{C}}+4 \ell_{\mathcal{C}}^{2}\right)^{2}\right)}{4\left(C_{\rho}+2 \ell_{\mathcal{C}}\right)^{8}} \\
Q_{\gamma} & =\frac{36 \ell_{\mathcal{C}}^{8}\left(4 \ell_{\mathcal{C}}^{6}-\left(C_{\rho}^{2}+4 C_{\rho} \ell_{\mathcal{C}}+4 \ell_{\mathcal{C}}^{2}\right)^{2}\right)}{\left(C_{\rho}+2 \ell_{\mathcal{C}}\right)^{8}} \\
\left(\Theta_{\mathrm{I}}\right)^{2} & =-\ell_{\mathcal{C}}^{2} Q_{\gamma} .
\end{aligned}
$$

By Corollary 1, conditions (82) give an invariant characterization of the equivalence class of metrics equivalent to the Van den Bergh metric. 


\section{Conclusions}

Considering metrics with two commuting Killing vectors, referred to as $G_{2} I$-metrics, we introduced scalar differential invariants of the first and second order with respect to the pseudogroup of transformations preserving the Riemannian submersion structure. The set of (semi-)invariants with tractable coordinate expressions is sufficient for the solution of the equivalence problem in the generic case. The next goal is to look for relations satisfied by known metrics or classes thereof. By computing all metrics satisfying these relations, one can, in principle, either extend the set of known solutions or prove an invariant characterization of a class of metrics in the spirit of [12]. As an example, we extended the Kundu class, defined by $\ell_{\mathcal{C}}=0$, to the $\Lambda$-vacuum case.

\section{References}

1. Alekseev, G.A.: Thirty years of studies of integrable reductions of Einstein's field equations. In: Damour, T., Jantzen, R.T. (eds.) The Twelfth Marcel Grossmann Meeting, pp. 645-666. World Scientific, Singapore (2012)

2. Alekseevsky, D.V., Vinogradov, A.M., Lychagin, V.V.: Basic Ideas and Concepts of Differential Geometry, Encyclopaedia Mathematics Science, Vol. 28 (Springer, Berlin, 1991)

3. Åman, J.E., Karlhede, A.: An algorithmic classification of geometries in general relativity. In: Proceedings of the Fourth ACM Symposium on Symbolic and Algebraic Computation, Snowbird, Utah, USA (ACM), pp. 79-84 (1981)

4. Belinskiŭ, V.A., Zakharov, V.E.: Integration of the Einstein equations by means of the inverse scattering problem. Sov. Phys. JETP 75(6), 1955-1971 (1978)

5. Besse, A.L.: Einstein Manifolds. Springer, Berlin (1987)

6. Bradley, M., Marklund, M.: Finding solutions to Einstein's equations in terms of invariant objects. Class. Quantum Gravity 13, 3021-3037 (1996)

7. Carminati, J., McLenaghan, R.G.: Algebraic invariants of the Riemann tensor in a four-dimensional Lorentzian space. J. Math. Phys. 32, 3135-3140 (1991)

8. Carter, B.: Killing horizons and orthogonally transitive groups in space-time. J. Math. Phys. 10, 70-81 (1969)

9. Coley, A., Hervik, S., Pelavas, N.: Spacetimes characterized by their scalar curvature invariants. Class. Quantum Gravity 26, 025013 (2009). (33pp)

10. Cosgrove, C.M.: A new formulation of the field equations for the stationary axisymmetric gravitational field: I. General theory. J. Phys. A Math. Gen. 11, 2389-2404 (1978)

11. Cosgrove, C.M.: A new formulation of the field equations for the stationary axisymmetric gravitational field: II. Separable solutions. J. Phys. A Math. Gen. 11, 2405-2430 (1978)

12. Ferrando, J.J., Sáez, J.A.: An intrinsic characterization of the Kerr metric. Class. Quantum Gravity 26, 075013 (2009). (13pp)

13. Gaffet, B.: The Einstein equations with two commuting Killing vectors. Class. Quantum Gravity 7, 2017-2044 (1990)

14. Geroch, R.: A method for generating solutions of Einstein's equations. J. Math. Phys. 12, 918-924 (1971)

15. Geroch, R.: A method for generating new solutions of Einstein's equations. II. J. Math. Phys. 13, 394-404 (1972)

16. Karlhede, A.: A review of the geometrical equivalence of metrics in general relativity. Gen. Rel. Gravit. 12, 693-707 (1980)

17. Karlhede, A.: The equivalence problem. Gen. Rel. Gravit. 38, 1109-1114 (2006)

18. Karlhede, A., MacCallum, M.A.H.: On determining the isometry group of a Riemannian space. Gen. Rel. Gravit. 14, 673-682 (1982)

19. Klein, C., Richter, O.: Ernst Equation and Riemann Surfaces. Springer, Berlin (2005)

20. Kolassis, C.A., Santos, N.O.: Spacetimes with a preferred null direction and a two-dimensional group of isometries: the null dust case. Class. Quantum Gravity 4, 599-618 (1987)

21. Kundu, P.: Class of "noncanonical" vacuum metrics with two commuting Killing vectors. Phys. Rev. Lett. 42, 416-417 (1979)

22. Lewis, T.: Some special solutions of the equations of axially symmetric gravitational fields. Proc. R. Soc. Lond. A 136, 176-192 (1932) 
23. Lychagin, V., Yumaguzhin, V.: Differential invariants and exact solutions of the Einstein-Maxwell equation. Anal. Math. Phys. 7, 19-29 (2017)

24. Lychagin, V., Yumaguzhin, V.: Differential invariants and exact solutions of the Einstein equations. Anal. Math. Phys. 7, 107-115 (2017)

25. Marvan, M., Stolín, O.: On local equivalence problem of spacetimes with two orthogonally transitive commuting Killing fields. J. Math. Phys. 49(2), 022503 (2008). 17 pp

26. Milson, R., McNutt, D., Coley, A.: Invariant classification of vacuum pp-waves. J. Math. Phys. 54, 022502 (2013)

27. Olver, P.J.: Equivalence, Invariants and Symmetry. Cambridge University Press, New York (1995)

28. O’Neill, B.: The fundamental equations of a submersion. Mich. Math. J. 13, 459-469 (1966)

29. Ovsiannikov, L.V.: Group Analysis of Differential Equations. Academic Press, New York (1982)

30. Petrov, A.Z.: Einstein Spaces. Pergamon, New York (1969)

31. Papapetrou, A.: Champs gravitationnels stationnaires à symétrie axiale. Ann. Inst. H. Poincaré A 4(2), 83-105 (1966)

32. Pollney, D., Skea, J.E.F., d'Inverno, R.A.: Classifying geometries in general relativity: III. Classification in practice. Class. Quantum Gravity 17, 2885-2902 (2000)

33. Pravda, V., Pravdová, A., Coley, A., Milson, R.: All spacetimes with vanishing curvature invariants. Class. Quantum Gravity 19, 6213-6236 (2002)

34. Skea, J.E.F.: A spacetime whose invariant classification requires the fifth covariant derivative of the Riemann tensor. Class. Quantum Gravity 17, L69-L74 (2000)

35. Stephani, H., Kramer, D., MacCallum, M., Hoenselaers, C., Herlt, E.: Exact Solutions of Einstein's Field Equations, 2nd edn. Cambridge University Press, Cambridge (2003)

36. Verdaguer, E.: Soliton solutions in spacetime with two spacelike Killing fields. Phys. Rep. 229(1-2), 1-80 (1993)

37. Van den Bergh, N.: A class of inhomogeneous cosmological models with separable metrics. Class. Quantum Gravity 5, 167-177 (1988)

38. Whelan, J.T., Romano, J.D.: Quasistationary binary inspiral. I. Einstein equations for the two Killing vector spacetime. Phys. Rev. D 60, 084009 (1999)

39. Lim, W.C.: Non-orthogonally transitive G2 spike solution. Class. Quantum Gravity 32, 162001 (2015)

40. Żorawski, K.: On deformation invariants. An application of Lie's theory of groups. Acta Math. 16, 1-64 (1892) (in German)

Publisher's Note Springer Nature remains neutral with regard to jurisdictional claims in published maps and institutional affiliations. 